\title{
Peptidergic Modulation of Intrathalamic Circuit Activity In Vitro: Actions of Cholecystokinin
}

\author{
Charles L. Cox, John R. Huguenard, and David A. Prince \\ Department of Neurology and Neurological Sciences, Stanford University Medical Center, Stanford, California 94305
}

\begin{abstract}
Cholecystokinin (CCK)-mediated actions on intrathalamic rhythmic activities were examined in an in vitro rat thalamic slice preparation. Single electrical stimuli in the thalamic reticular nucleus (nRt) evoked rhythmic activity (1-15 sec duration) in $\mathrm{nRt}$ and the adjacent ventrobasal nucleus (VB). Low CCK concentrations (20-50 nM) suppressed rhythmic oscillations in $43 \%$ of experiments but prolonged such activities in the remaining slices. Higher CCK concentrations (100-400 nM) had a predominantly antioscillatory effect.

Suppression of oscillations was associated with a relatively large membrane depolarization of nRt neurons that changed their firing mode from phasic (burst) to tonic (single-spike) output. This decreased burst discharge of $n R t$ neurons during CCK application reduced inhibitory drive onto VB neurons from multiple peaked inhibitory postsynaptic currents (IPSCs) to single peaked inhibitory events. We hypothesize that suppres-
\end{abstract}

sion of inhibitory drive onto VB neurons decreases their probability of burst output, which, together with a reduction of $\mathrm{nRt}$ burst output, dampens the oscillatory activity.

Low CCK concentrations, which produced little or no depolarization of $\mathrm{nRt}$ neurons, did not alter the firing mode of the $\mathrm{nRt}$ neurons. However, the probability of burst output from nRt neurons in response to subthreshold stimuli was increased in low CCK concentrations, presumably leading to an increase in the number of nRt neurons participating in the rhythmic activity. Our findings suggest that the neuropeptide CCK, by altering the firing characteristics of nRt neurons, has powerful modulatory effects on intrathalamic rhythms; the ultimate action was dependent on CCK concentration and resting state of these cells.

Key words: cholecystokinin; thalamic reticular nucleus; oscillations; ventrobasal thalamus; neuropeptides; neuromodulation
Rhythmic activities in thalamocortical circuits occur during sensory processing, slow-wave sleep, and pathophysiological conditions such as petit mal epilepsy (Williams, 1953; Hubel, 1960; Domich et al., 1986; Steriade and Llinás, 1988; Gray and Singer, 1989). The reciprocal synaptic connectivity between the thalamic reticular nucleus (nRt), or the analogous perigeniculate nucleus, and adjacent thalamic relay nuclei, in conjunction with the intrinsic properties of thalamic neurons, can lead to generation of these neuronal oscillations (Steriade and Llinás, 1988; Steriade et al., 1993; von Krosigk et al., 1993; Huguenard and Prince, 1994; Warren et al., 1994). Certain neuromodulators, such as acetylcholine (ACh), norepinephrine (NE), and serotonin (5-HT), which arise from brainstem nuclei, can alter the excitability of thalamic neurons and modify the rhythmic activity (McCormick and Prince, 1987; McCormick and Prince, 1988; Hu et al., 1989a,b; McCormick and Wang, 1991). Little is known regarding the intrinsic cellular mechanisms that control the initiation and cessation of these rhythms (see Bal and McCormick, 1993) or their regulation by substances endogenous to intrathalamic circuits.

The firing mode of nRt neurons is important for the maintenance of thalamic rhythmic activity (Steriade and Deschênes, 1984; Steriade and Llinás, 1988; von Krosigk et al., 1993; Huguenard and Prince, 1994; Bal et al., 1995). Burst discharges in these cells can produce powerful IPSPs in thalamocortical relay (TC) neurons, that in turn activate a low-threshold $\mathrm{Ca}^{2+}$ spike (LTS),

Received July 19, 1996; revised Oct. 3, 1996; accepted Oct. 8, 1996.

This research was supported by National Institutes of Health Grants NS06477 and NS07280 and the Morris and Pimley Research Funds.

Correspondence should be addressed to Dr. Charles L. Cox, Department of Neurology and Neurological Sciences, Room M016, Stanford University Medical Center, Stanford, CA 94305.

Copyright (C) 1996 Society for Neuroscience $0270-6474 / 96 / 170070-13 \$ 05.00 / 0$ which generates bursts of $\mathrm{Na}^{+}$-dependent action potentials (Deschênes et al., 1984; Jahnsen and Llinás, 1984; Steriade and Deschênes, 1984; von Krosigk et al., 1993). Thalamocortical cells form excitatory synapses on $\mathrm{nRt}$ neurons and reexcite them to produce burst discharge, continuing the cycle. Thus, burst discharge from neurons in both nuclei are critical for the maintenance of the intrathalamic rhythmic activity.

Cholecystokinin (CCK) is one of many neuropeptides localized within thalamic neurons (Hunt et al., 1987; Ogawa et al., 1989; Schiffmann and Vanderhaeghen, 1991; Battaglia et al., 1992; Burgunder and Young, 1992). CCK is present within TC and corticothalamic neurons (Ingram et al., 1989; Burgunder and Young, 1990; Battaglia et al., 1992; Senatorov et al., 1995), receptors for this peptide are located in the thalamus (Zarbin et al., 1983; Pelaprat et al., 1987), and peripheral stimulation can increase CCK levels in the thalamus (Xu et al., 1990). In other regions, neuropeptides are colocalized with "classical" neurotransmitters (Lundberg and Hökfelt, 1983; Hendry et al., 1984; Hökfelt et al., 1986), can be differentially released by activity-dependent processes (Bartfai et al., 1988), and can modulate neuronal excitability (Kupfermann, 1991). CCK can modify single-unit activity in nRt and the lateral geniculate nucleus (Albrecht et al., 1994; Davidowa et al., 1995; Cox et al., 1995a). Intracellular recordings of nRt neurons reveal that CCK decreases a leak potassium conductance $\left(K_{\text {leak }}\right)$, resulting in a longlasting membrane depolarization (Cox et al., 1995a). Because CCK is endogenous to the thalamocortical circuit and produces enduring modifications of neuronal excitability, this compound may serve as an intrinsic, long-term regulator of intrathalamic rhythms.

Rat thalamic slices maintained in vitro possess sufficient connectivity to generate slow intrathalamic rhythms resembling those observed during slow-wave sleep and petit mal seizures (Hugue- 


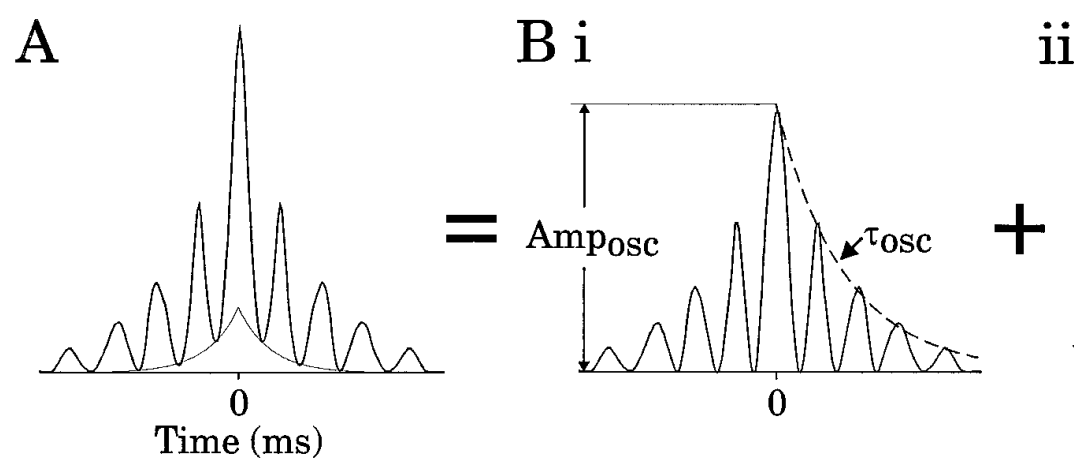

ii

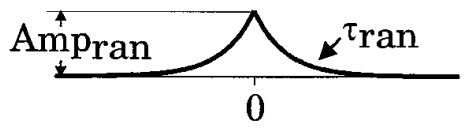

Figure 1. Components of autocorrelograms. Schematic of representative autocorrelogram constructed from extracellular multiple-unit data. The plot consists of distinct peaks with nonzero valleys. The phasic synchronous oscillatory activity $(B i)$ was separated from the nonzero valleys, which represent nonphasic activity $(B i i)$. The area under the valleys represents residual nonsynchronous discharge activity $($ Bii $)$, and the decay of this activity $\left(\tau_{\text {ran }}\right)$ is fit by a single exponential. The decay of the oscillation $\left(\tau_{\text {osc }}\right)$ is calculated by a single-exponential fit of the oscillatory peaks $(B i$, dashed line $)$. The oscillation amplitude $\left(A m p_{\text {osc }}\right)$ corresponds to the height of the central peak of the autocorrelogram.

nard and Prince, 1994). We have investigated the actions of CCK on intrathalamic rhythms using this preparation. Because CCK induces membrane depolarizations (Cox et al., 1995a) that could alter the firing mode of nRt neurons from bursting to single-spike in a manner similar to that reported for NE and 5-HT (McCormick and Prince, 1988; McCormick and Wang, 1991), we hypothesized that this peptide would suppress the intrathalamic rhythms. Our results indicate that CCK has dual effects on the phasic intrathalamic activities. At low concentrations, CCK can either facilitate or suppress the rhythmic activity, whereas at higher concentrations, CCK suppresses this activity. Some of these findings have been presented in abstract form (Cox et al., 1995b).

\section{MATERIALS AND METHODS}

General procedures were similar to those described previously (Cox et al., 1995a). Briefly, young Sprague Dawly rats (9-21 d) were deeply anesthetized with pentobarbital sodium $(55 \mathrm{mg} / \mathrm{kg})$ and decapitated. The brains were quickly removed and placed into cold, oxygenated slicing medium $\left(\sim 4^{\circ} \mathrm{C}\right.$ ). The slicing medium contained (in $\mathrm{mM}$ ): $2.5 \mathrm{KCl}, 1.25 \mathrm{NaH}_{2} \mathrm{PO}_{4}$, $10.0 \mathrm{MgCl}_{2}, 0.5 \mathrm{CaCl}_{2}, 26.0 \mathrm{NaHCO}_{3}, 11.0$ glucose, and 234.0 sucrose. Tissue slices $(300-450 \mu \mathrm{m})$ were cut in the horizontal plane using a vibratome (Ted Pella, Inc., St. Louis, MO), transferred to a holding chamber, and incubated $\left(30^{\circ} \mathrm{C}\right)$ for at least $2 \mathrm{hr}$ before recording. Individual slices were then transferred to a recording chamber and allowed to equilibrate for at least $30 \mathrm{~min}$ before recording. Slices were minimally submerged and continuously superfused with oxygenated physiological saline at the rate of $1.3 \mathrm{ml} / \mathrm{min}$ in the chamber used for extracellular and "blind" whole cell recordings (Blanton et al., 1989), or $4.0 \mathrm{ml} / \mathrm{min}$ in the chamber used for "visualized slice" recordings. The majority of recordings were done at a temperature of $34 \pm 1^{\circ} \mathrm{C}$, although, as noted in Results, some recordings in the visualized slice apparatus were at room temperature $\left(23^{\circ} \mathrm{C}\right)$. The physiological solution used in the intracellular experiments contained (in mM): $126.0 \mathrm{NaCl}, 2.5 \mathrm{KCl}, 1.25 \mathrm{NaH}_{2} \mathrm{PO}_{4}, 2.0$ $\mathrm{MgCl}_{2}, 2.0 \mathrm{CaCl}_{2}, 26.0 \mathrm{NaHCO}_{3}$, and 10.0 glucose. These solutions were gassed with $95 \% \mathrm{O}_{2} / 5 \% \mathrm{CO}_{2}$ to a final $\mathrm{pH}$ of 7.4 .

Recording procedure. Whole-cell recordings were obtained using either blind (Blanton et al., 1989) or visualized slice patch techniques (Edwards et al., 1989; Stuart et al., 1993). Recording pipettes were pulled from 1.5 $\mathrm{mm}$ outer diameter capillary tubing using a two-stage pipette puller (Narishige PP-83) and had tip resistances of 4-6 M $\Omega$ when filled with the intracellular solutions below. The intracellular solution used in the majority of recordings contained (in $\mathrm{mm}$ ): $117 \mathrm{~K}$-gluconate, $13 \mathrm{KCl}, 1.0$ $\mathrm{MgCl}_{2}, 0.07 \mathrm{CaCl}_{2}, 0.1$ EGTA, 10.0 HEPES, $2.0 \mathrm{Na}_{2}$-ATP, and 0.4 Na-GTP. In some experiments, Cs-gluconate and $\mathrm{CsCl}$ were substituted for K-gluconate and $\mathrm{KCl}$, respectively. The $\mathrm{pH}$ of the solution was adjusted to 7.3 using $\mathrm{KOH}$ or $\mathrm{CsOH}$, and osmolality was adjusted to 280 mOsm with distilled water.

Techniques used for obtaining recordings with the blind patch configuration were similar to those described earlier (Cox et al., 1995a). For recordings obtained using the visualized slice preparation, individual slices were transferred to a submersion-type recording chamber on a modified microscope stage. A low-power objective $(2.5 \times)$ was used to identify the various thalamic nuclei, and a high-power water immersion objective $(40 \times)$ with Nomarski optics and infrared video was used to visualize individual neurons. The access resistance was typically 10-40 $\mathrm{M} \Omega$ in the blind preparation and 5-15 $\mathrm{M} \Omega$ in the visualized preparation.

An Axoclamp2A amplifier (Axon Instruments, Foster City, CA) was used in bridge mode for voltage recordings and discontinuous singleelectrode voltage-clamp mode for current recordings. In current-clamp recordings, an active bridge circuit was adjusted to balance the drop in potential produced by passing current through the recording electrode. In voltage-clamp recordings, switching frequencies ranged from 2.5 to $4 \mathrm{kHz}$ with a gain of $150-800 \mathrm{pA} / \mathrm{mV}$, and the headstage was monitored continually to ensure that the current transient had completely decayed before voltage measurements. Voltage-clamp recordings were limited to neurons that had a stable access resistance of $<25 \mathrm{M} \Omega$. Current and voltage protocols were generated using PCLAMP software (Axon Instruments), and data were stored on an IBM PC-compatible computer and magnetic tape (Neurodata).

Extracellular multiple-unit activities were recorded using monopolar tungsten electrodes (0.2-2 M $\Omega$; Frederick Haer, Brunswick, ME) and a Grass amplifier (bandwidth, $0.3-10 \mathrm{kHz}$ ). All data were digitized (1-2 $\mathrm{kHz}$ ) and stored using Axotape software (Axon Instruments). Monopolar or bipolar extracellular stimuli were delivered through sharpened tungsten electrodes.

Drugs. Concentrated stock solutions of CCK8S $(1.0 \mathrm{~mm})$ were prepared in $0.1 \mathrm{M} \mathrm{NH}_{4} \mathrm{HCO}_{3}$ and diluted in physiological saline to a final concentration of $0.01-10 \mu \mathrm{M}$. CCK8S was bath-applied in the majority of experiments. In some blind slice experiments, CCK8S was applied by injecting a bolus into the flow line of the chamber over 10-15 sec using a motorized pump. In these instances, based on the rate of CCK8S injection and the rate of chamber perfusion, the final bath concentration of CCK was estimated to be about one-fourth of the concentration introduced in the flow line (Cox et al., 1995a).

Concentrated stock solutions of antagonists were diluted to a final concentration in physiological solution and applied by bath. CCK8S was purchased from Peninsula Laboratories (Belmont, CA), CNQX from Tocris Cookson (St. Louis, MO), and AP-5 from RBI (Natick, MA). CGP-35348 was a gift from CIBA-Geigy (Basel, Switzerland), and R(-)3-(2-carboxypiperazin-4-yl)-propyl-1-phosphonic acid (d-CPPene) from Sandoz (Berne, Switzerland). All remaining compounds were purchased from Sigma (St. Louis, MO).

Analysis of intrathalamic oscillations. To quantify the degree of synchrony and the duration of the intrathalamic oscillations, autocorrelograms were constructed from the extracelluar multiple-unit data. A software Schmidt trigger was used to detect events, and the autocorrelograms were calculated over a period of $4-8 \mathrm{sec}$ with a bin size of $8-10 \mathrm{~ms}$. Autocorrelograms typically consisted of peaks of correlated phasic activity superimposed on random unit activity, as suggested by the nonzero counts in the interpeak valleys (Fig. $1 A$ ). We divided the functions into two components: the phasic oscillatory peaks (Fig. $1 \mathrm{Bi}$ ) and the nonphasic random component that represented random unit activity (Fig. 1Bii). 
The random component was typically fit by a single exponential and could be subtracted from the overall auto correlogram. The oscillatory activity in the autocorrelograms was quantified by four measures: number of peaks; time constant of oscillation decay $\left(\tau_{\mathrm{osc}}\right)$; oscillation amplitude $\left(A m p_{o s c}\right)$; and the period of oscillation. The $\tau_{\text {osc }}$ was fitted by single exponential function and served as an indication of the duration of the oscillatory activity. This is a measure of the ability of the nRt-VB slice to self-sustain rhythmic discharges. The number of autocorrelogram peaks was used as a secondary measure of oscillation duration. The oscillation period reflects the principle frequency of the activity. Amp osc $_{\text {represents }}$ the peak phasic activity and synchrony after subtraction of the random nonphasic component. Alterations in $\mathrm{Amp}_{\text {osc }}$ suggest a change in the number of units participating in the phasic activity. The enhanced $\mathrm{Amp}_{\mathrm{osc}}$ in the extracellular records was presumably a result of recruitment of additional neuronal units into phasic activity (see Fig. 9), and perhaps an increase in synchrony of cell discharge. Amp ${ }_{\text {osc }}$ and $\tau_{\text {osc }}$ were calculated only when the overall oscillation consisted of $>2$ cycles. In those slices in which CCK completely suppressed the rhythmic activity, only the number of peaks was quantified.

\section{RESULTS}

\section{Intrathalamic oscillations}

As shown previously, the reciprocal synaptic connectivity between $\mathrm{nRt}$ and $\mathrm{VB}$ is preserved in the in vitro thalamic preparation and supports intrathalamic rhythmic activities (Huguenard and Prince, 1994; Warren et al., 1994). Electrical stimulation of internal capsule (IC) or nRt (Fig. $2 A$ ) produced 2-15 regularly reoccurring excitatory postsynaptic potentials (EPSPs) that had an average frequency of $3.1 \pm 0.6 \mathrm{~Hz}(n=12$; e.g., Fig. $2 B, n R t)$. These EPSPs could lead to bursts of $\mathrm{Na}^{+}$-dependent action potentials in nRt neurons (Fig. $2 B, *$ ). The complementary oscillatory activity in VB consisted of rhythmic IPSPs (Fig. 2B) that could lead to rebound burst discharge after membrane repolarization $(2 B, \boldsymbol{\nabla})$. A similar oscillatory activity that averaged $2.7 \pm$ $0.3 \mathrm{~Hz}(n=12$ slices) could also be detected in extracellular multiple-unit recordings (e.g., Fig. 2C); these recordings were used to monitor rhythmic activities in large neuronal populations.

In most slices used for extracellular recordings, the oscillatory response evoked in normal physiological solution lasted 1-2 cycles but was quite variable between slices. Likely factors in slice-toslice variations in rhythmic activity were differences in the extent of functional connectivity between nRt and VB and in the mean resting potentials of the neurons. The strength and duration of the rhythmic activities could be increased by the addition of the $\mathrm{GABA}_{\mathrm{A}}$-receptor antagonist bicuculline methiodide (BMI, Huguenard and Prince, 1994) and by reducing extracellular $\mathrm{Mg}^{2+}$ concentrations. In all extracellular experiments, we used a modified physiological solution containing $0.2 \mathrm{mM} \mathrm{Mg}^{2+}$ and $1 \mu \mathrm{M} \mathrm{BMI}$ (as noted in figure legends) to enhance the network oscillation and reduce the variability between slices. Under these conditions, oscillations lasting 1-4 sec could be evoked regularly in most slices. In a few experiments, higher BMI concentrations ( 2 or 10 $\mu \mathrm{M})$ were necessary to achieve similar duration oscillations. Once these modifications in the perfusate were made, the stimulievoked oscillations were remarkably stable in a given slice or neuron (e.g., see Figs. 4ii, 5ii, Pre-drug), thus providing a stationary baseline before CCK application. These manipulations in the perfusate did not alter the basic configuration of the $2-5 \mathrm{~Hz}$ rhythm, suggesting that essential synaptic and intrinsic oscillatory mechanisms were still effective. It is important to note that intracellular recordings of rhythmic EPSPs indicated that the $2-5 \mathrm{~Hz}$ oscillations occurred either in normal physiological solution $(n=$ 8; e.g., Fig. $7 B)$ or in the presence of BMI $(n=3$; e.g., Fig. $7 A)$.

Low BMI concentrations $(1-10 \mu \mathrm{M})$ enhanced the $2-5 \mathrm{~Hz}$ network oscillation (Figs. 2C, 3A; Huguenard and Prince, 1994).
A.

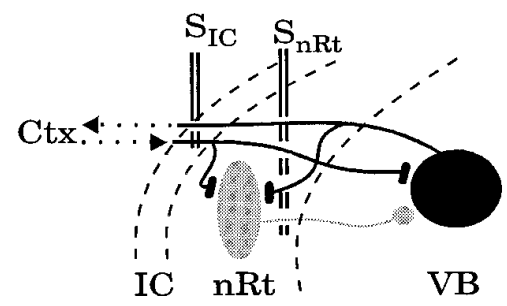

B.

nRt
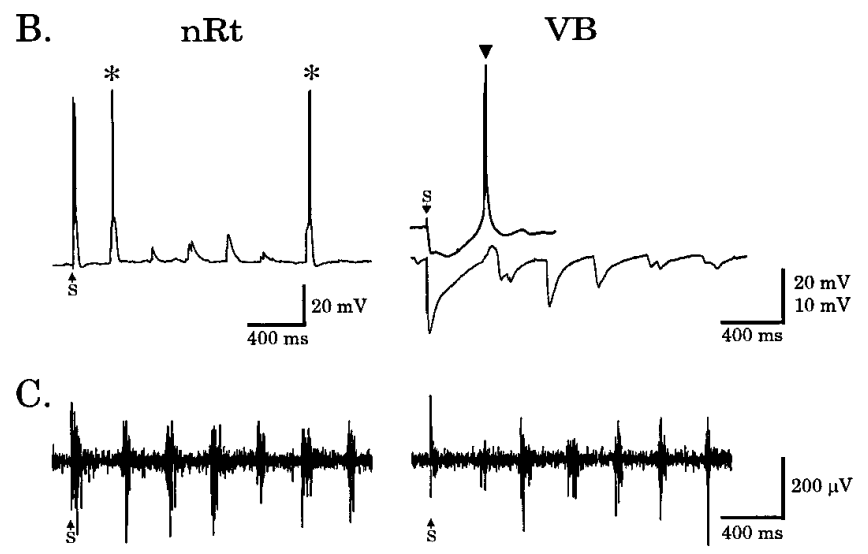

Figure 2. Intrathalamic oscillations recorded in nRt and VB. $A$, Schematic of intrathalamic circuit in thalamic slice preparation. nRt provides inhibitory innervation of VB that in turn forms excitatory synapses on $\mathrm{nRt}$ cells. Monopolar stimuli were delivered to either nRt $\left(S_{n R t}\right)$ or internal capsule $\left(S_{I C}\right)$. Ctx, Cortex. $B$, Intracellular current-clamp recordings from $\mathrm{nRt}$ and VB neurons illustrating the rhythmic synaptic activity evoked by single extracellular stimuli $(S)$. $n R t$, Responses in an nRt cell consist of rhythmic EPSPs $(3.8 \mathrm{~Hz})$ that can lead to burst discharge (asterisks). VB, Similar stimuli evoke rhythmic IPSPs $(2.9 \mathrm{~Hz})$ in a VB neuron (bottom trace). The top trace corresponds to a recording from a different $\mathrm{VB}$ neuron and illustrates that a burst discharge $(\boldsymbol{\nabla})$ can occur during membrane repolarization after the IPSPs. The $20 \mathrm{mV}$ calibration refers to the top trace, and the $10 \mathrm{mV}$ calibration to the bottom trace. These recordings were made in standard physiological solution with no addition of BMI. The $\mathrm{K}^{+}$-containing intracellular solution was used in these recordings, as well as those illustrated in Figures $7-9 . V_{\mathrm{m}},-64$ and $-58 \mathrm{mV}$ for $\mathrm{nRt}$ and VB neuron, respectively. $C$, Simultaneous extracellular recordings from $\mathrm{nRt}$ and $\mathrm{VB}$ reveal rhythmic multiple-unit discharge $(3.4 \mathrm{~Hz})$ in response to extracellular IC stimulation $(S)$. The extracellular solution for these recordings contained $0.2 \mathrm{mM} \mathrm{Mg}^{2+}$ and $10 \mu \mathrm{M}$ BMI.

Within individual slices, the concentration of BMI was correlated positively with the duration and synchronicity of the oscillations. As illustrated in Figure $3 A$, the oscillation duration and number of participating units in each cycle increased in higher BMI concentrations. Autocorrellograms were used to quantify the synchronicity and periodicity of the oscillatory activity (see Materials and Methods). In $10 \mu \mathrm{M}$ BMI (Fig. 3B, gray line), the number of peaks and peak amplitudes was greater than observed in $2 \mu \mathrm{M}$ (Fig. 3B, black line), and the period was increased from 322 to 389 ms but remained in the $2-5 \mathrm{~Hz}$ range. Furthermore, $A_{\mathrm{mp}}$ osc and $\tau_{\text {osc }}$ were increased by $160 \%$ and $30 \%$, respectively, in $10 \mu \mathrm{M}$ BMI. The rhythm was completely abolished by the $\mathrm{GABA}_{\mathrm{B}}$ antagonist CGP-35348 (Fig. 3A, $400 \mu \mathrm{M} ; n=3$ ), indicating the essential role of $\mathrm{GABA}_{\mathrm{B}}$-mediated synaptic responses in the network activity. This is illustrated clearly in the autocorrelogram by the nearly complete suppression of peaks (Fig. 3B, thick lines).

\section{CCK modulates intrathalamic oscillations}

In 6 of 14 experiments, low CCK concentrations (20-50 nм) suppressed the rhythmic activity (Fig. $4 A$ ). Typically, the baseline 
A

nRt

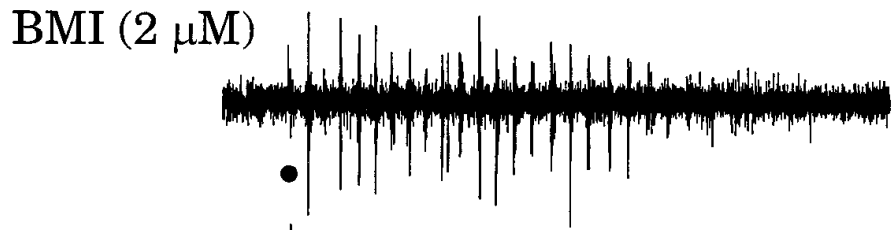

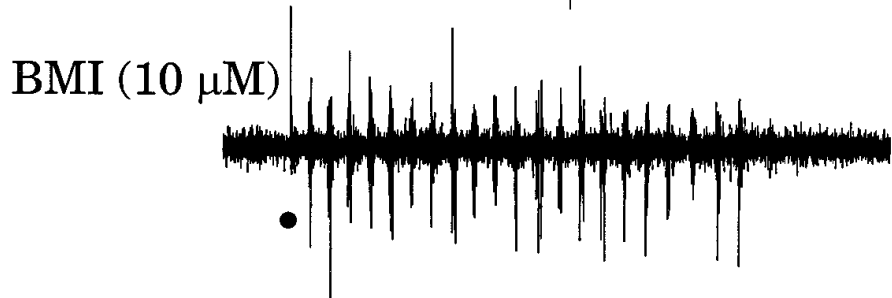

+CGP $35348(400 \mu \mathrm{M})$

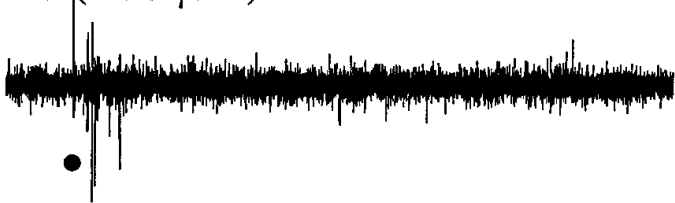

$\mathrm{B}$

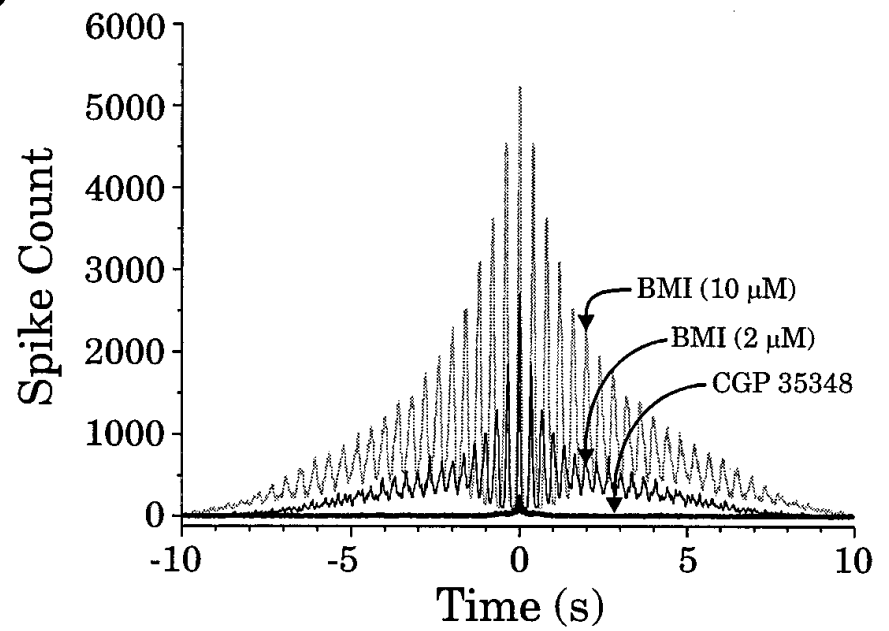

VB
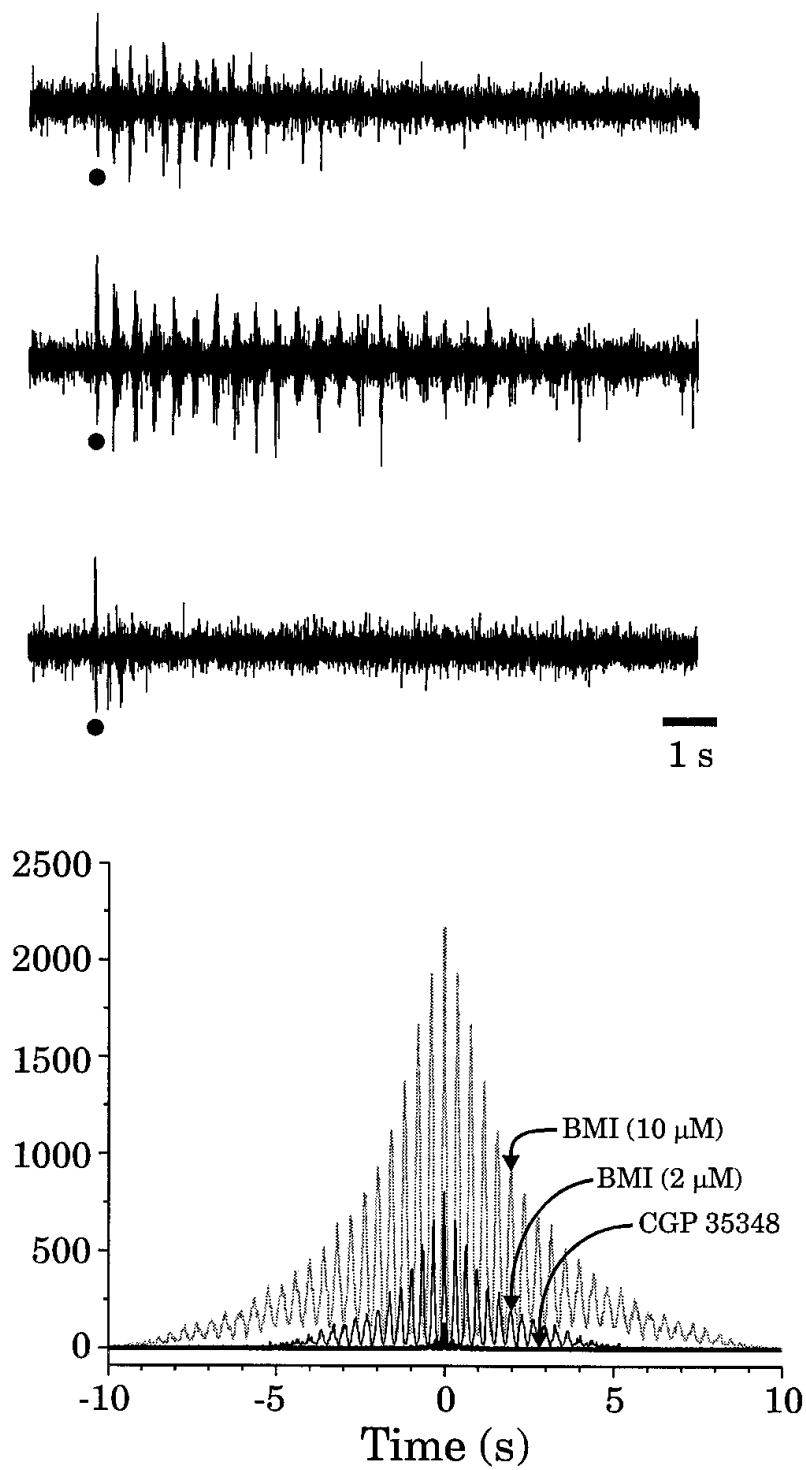

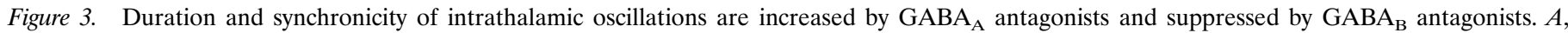

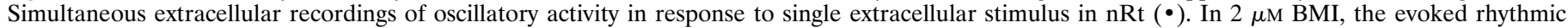

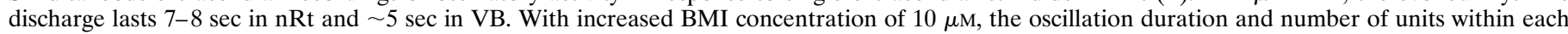

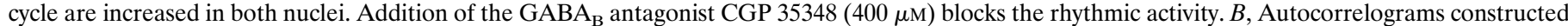

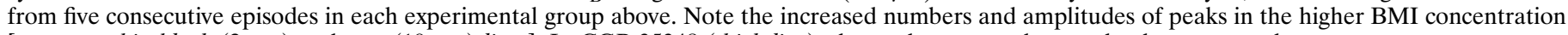
[compare thin black $(2 \mu \mathrm{M})$ and gray $(10 \mu \mathrm{M})$ lines]. In CGP 35348 (thick line), the peaks are nearly completely suppressed.

spike frequency was increased in nRt during CCK application, and subsequent stimulation evoked a robust discharge that lacked the phasic pattern observed in control conditions (Fig. 4Ai). Contour plots (Fig. 4Aii,Bii) illustrate the stability of the control response and the persistence and reversibility of the CCKmediated effects. In this slice, three cycles of oscillation were obtained consistently. CCK (20 nM) reversibly increased spontaneous firing and suppressed the rhythmic discharge. The autocorrelograms of Figure 4Aiii illustrate the CCK-induced decrease in oscillatory activity that was associated with complete suppression of distinct peaks and an overall increase in spike counts, effects consistent with increased nonphasic spike discharge.

It was surprising that in the remaining experiments (8 of 14),
CCK increased the duration of the rhythmic activity without altering the baseline discharge rate (Fig. 4Bi). The reversible increase in the duration of the oscillations is illustrated in the ratemeter output in Figure 4Bii (note different time bases in Figs. $4 A i, B i)$. In this slice, the oscillation increased from 10 cycles lasting approximately $3 \mathrm{sec}$ to 18 cycles lasting $>6 \mathrm{sec}$, an $80 \%$ increase in oscillatory activity (Fig. $4 B$ ). The autocorrelograms of Figure 4 Biii indicate the increased number and amplitude of peaks without alteration in the periodicity of the rhythm, suggesting an increase in the number of neurons participating in the phasic oscillatory activity.

In contrast to the anti- and pro-oscillatory actions observed in low CCK concentrations, the oscillatory activity was suppressed in 
A

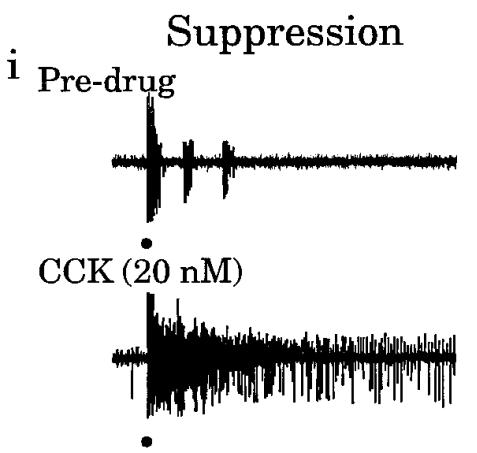

Wash

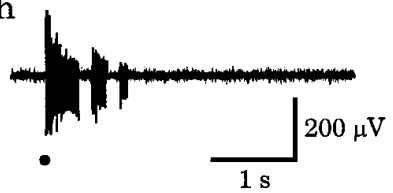

ii

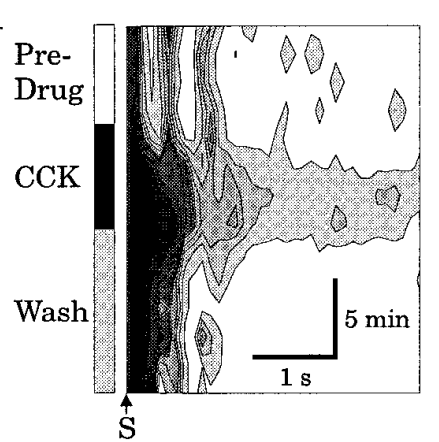

iii

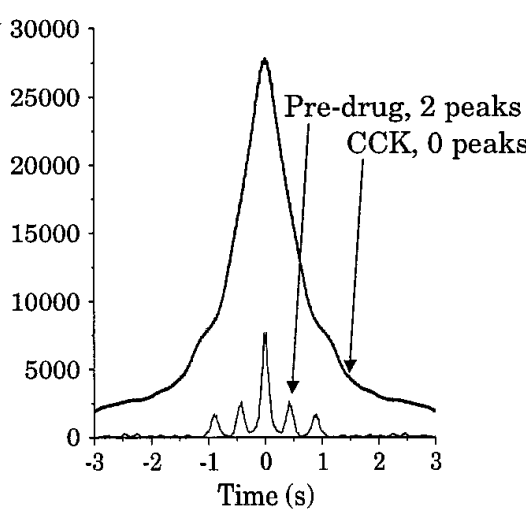

B

i

Pre-drug

\section{Facilitation}

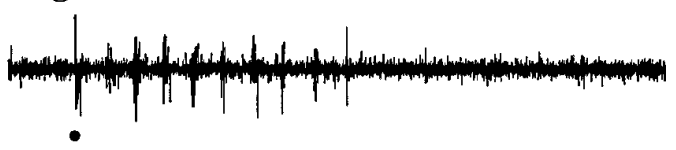

CCK (50 nM)

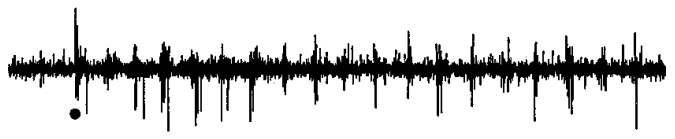

Wash

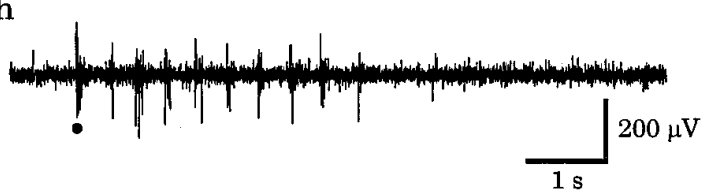

ii

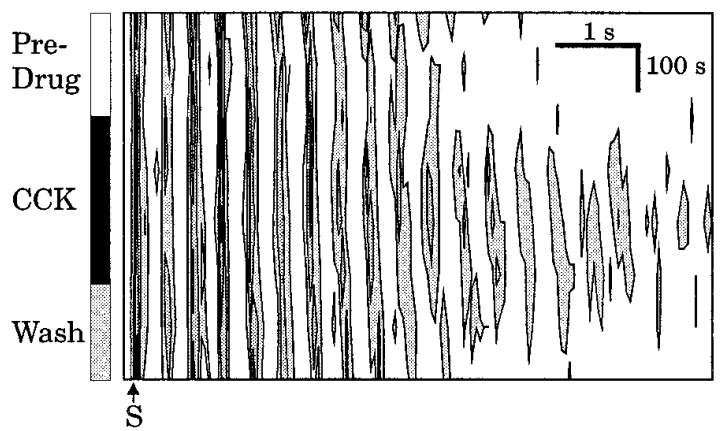

iii

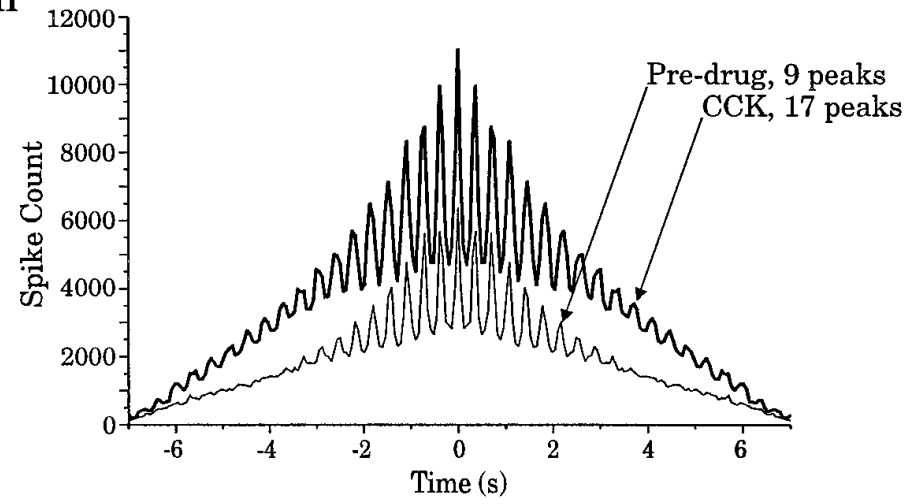

Figure 4. Low CCK concentrations either suppress or facilitate intrathalamic rhythms. A, Suppression. $i$, Extracellular multiple-unit recordings in nRt in control solution (Pre-drug), during $20 \mathrm{nM}$ CCK8S application, and after washout of the peptide. Stimulation of $\mathrm{nRt}(0.05 \mathrm{~Hz})$ in control solution containing $0.2 \mathrm{mM} \mathrm{Mg}^{2+}$ and $10 \mu \mathrm{M}$ BMI evokes three cycles of rhythmic discharge $(2.2 \mathrm{~Hz})$. Addition of $20 \mathrm{nM} \mathrm{CCK}$ attenuates the phasic response and increases background discharge and the duration of the initial response [CCK $(20 \mathrm{nM})]$. After washout of the peptide, the phasic response returns (Wash). Aii, Contour plot of same experiment. The ordinate represents the time course throughout the experiment (i.e., Pre-drug, CCK, Wash). The abscissa reflects the ratemeter output; darker levels correspond to higher firing frequencies. The phasic activity evoked by nt stimulation $(S)$ lasts for three cycles in the control condition (open bar, left). In the presence of CCK8S (solid bar), there is increased spike discharge that continues throughout the sampled period and a reduction in the distinct phasic cycles. During washout of the peptide (gray bar), the phasic activity returns toward baseline levels. The contour interval for this plot was 10 spikes. Note the decrease in phasic activity and increased discharge rate during CCK application. Aiii, Autocorrelograms of same experiment illustrating the overall increase in activity but loss of synchronized discharge in CCK (thick line). The overall increased amplitude and lack of distinguishable peaks is indicative of a nonphasic increase in spike discharge. $B$, Facilitation. $i$, In a different slice, stimulation of nRt evoked a phasic rhythmic discharge $(2.8 \mathrm{~Hz})$ in nRt lasting many seconds (Pre-drug). In this experiment, the extracellular perfusate contained $1 \mu \mathrm{M}$ BMI and 0.2 $\mathrm{mM} \mathrm{Mg}^{2+}$. CCK (50 nM), The duration of the rhythmic activity doubled and returned to baseline conditions after washout of CCK (Wash). Bii, Contour plot of the same experiment. This plot was constructed similar to Aii. Note the phasic activity evoked by the stimulus. During CCK application, the number of cycles significantly increased and returned to near baseline on washout. Biii, Autocorrelogram of the same experiment illustrates the synchronicity of the rhythmic activity. CCK (thick line) increased the amplitude as well as the number of peaks, indicative of the enhanced rhythmic activity. 
A

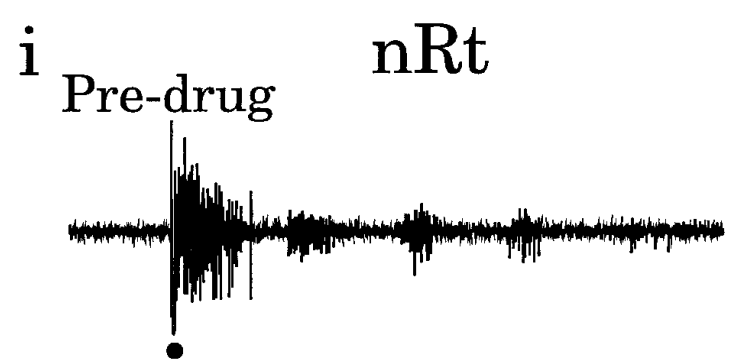

CCK (400 nM)

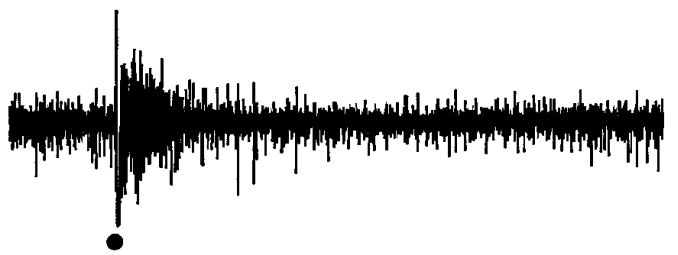

ii

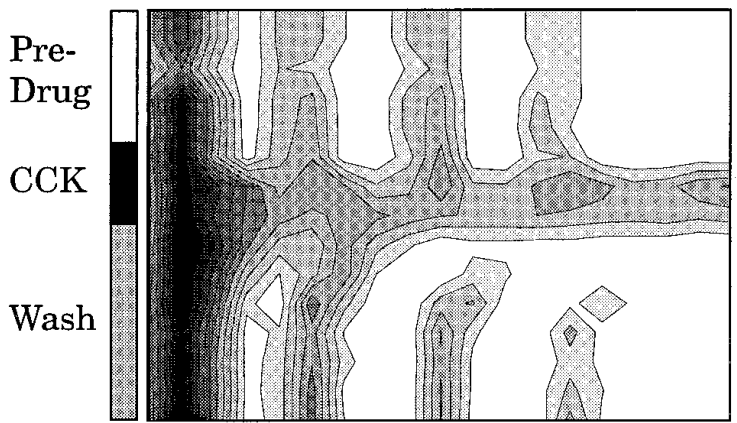

iii
$\mathrm{B}_{\mathrm{i}}$ i $\quad$ VB
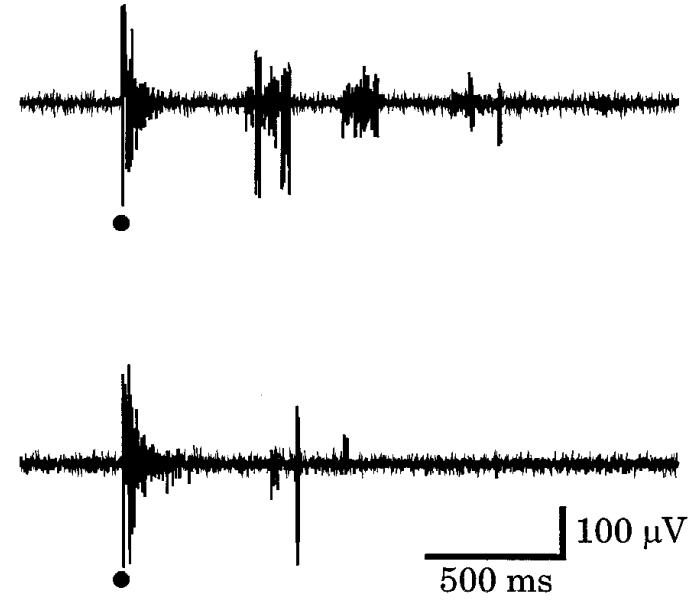

ii

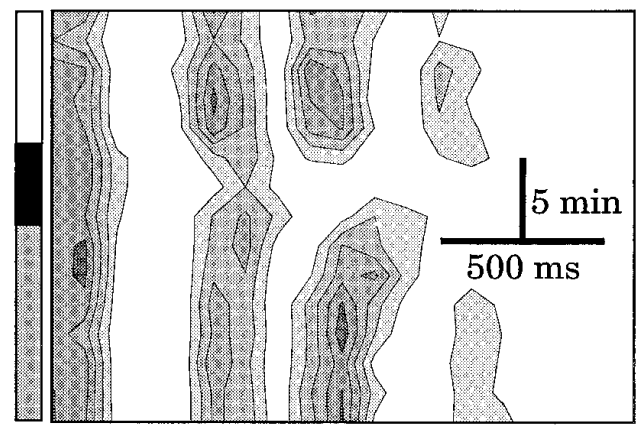

iii

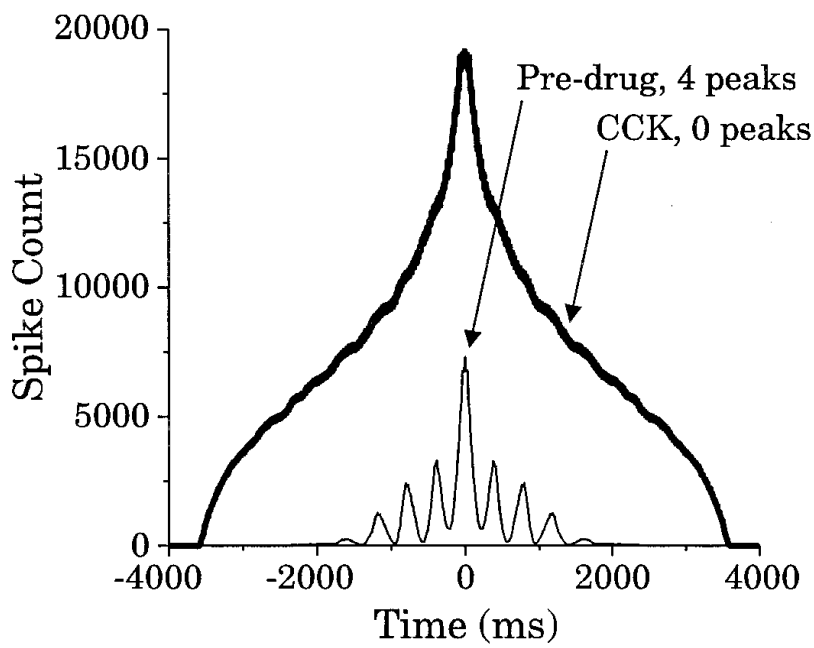

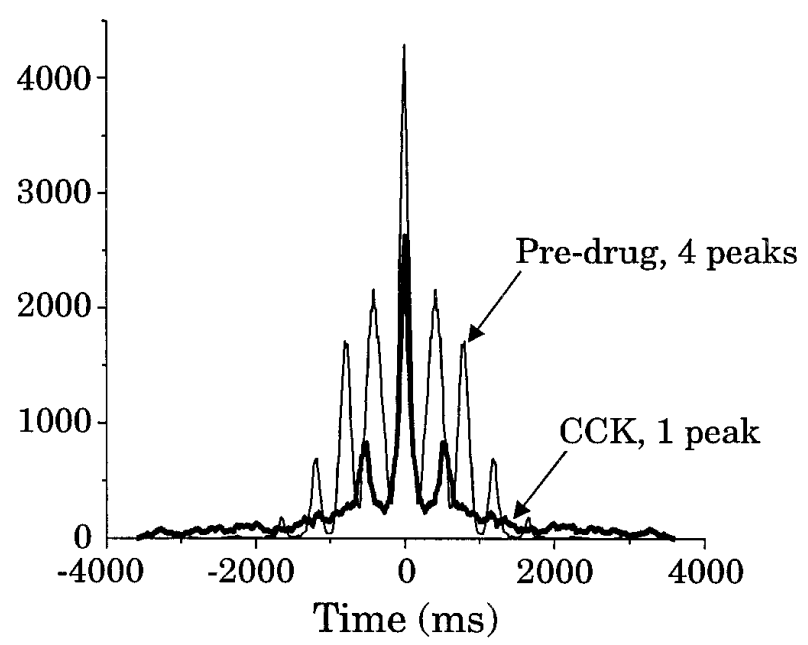

Figure 5. High CCK concentrations suppress intrathalamic rhythms. $A i, B i$, Stimulation of nRt evokes rhythmic discharge (2.5 Hz) in both nRt ( $A i)$ and VB (Bi). In CCK (400 nM), the rhythmic discharge in suppressed in both structures. In addition, there is an increased baseline discharge in nRt, consistent with a prolonged suprathreshold depolarization produced by the high concentration of CCK. The extracellular solution contained $10 \mu \mathrm{M}$ BMI and $0.2 \mathrm{mM}$ $\mathrm{Mg}^{2+}$. Aii, Bii, Contour plots illustrating the time course of the CCK effect in nRt (Aii) and VB (Bii). Four cycles are evoked in the pre-drug and wash periods. During CCK perfusion, continuous discharge occurs in nRt and a decrease in the number of cycles in VB. The contour interval for both plots was 20 spikes. See legend to Figure $4 B$ for further details of plot. Aiii, Biii, Autocorrelograms of unit activity in nRt (Aiii) and VB (Biii) in control conditions (thin line) and during application of $400 \mathrm{nM}$ CCK8S (thick line). Although the period of the remaining weak rhythmic activity (2 peaks) in this slice was slightly increased in CCK, there was not a significant change in the period for the population of slices (see Fig. 6 Bii). 
nRt

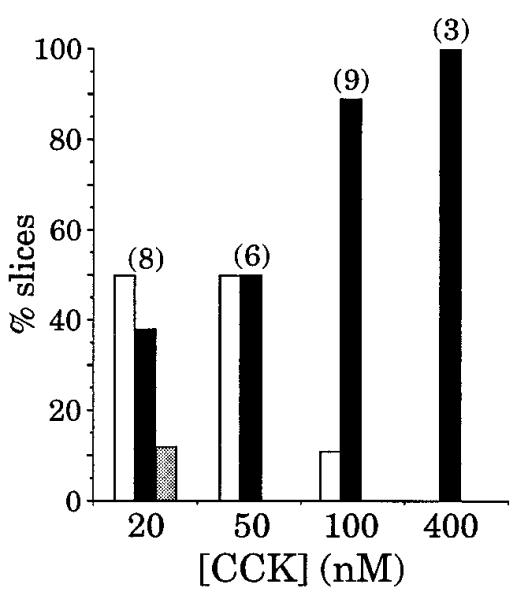

VB

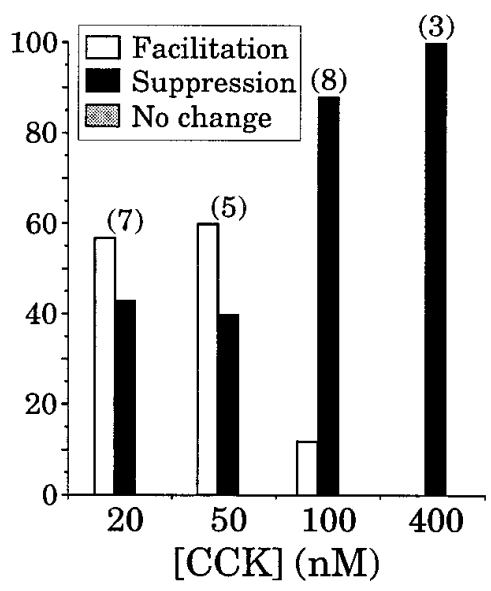

Figure 6. Summary of CCK actions on extracellularly recorded intrathalamic oscillations. $A$, Concentration dependence of CCK actions on thalamic rhythmic activity. The overall action of CCK (i.e., Facilitation, Suppression, No change) was determined by alterations of the number of peaks and Amp ${ }_{\text {osc }}$. Mixed effects (i.e., facilitation and suppression) were observed at lower CCK concentrations (20 and $50 \mathrm{nM}$ ); however, with increasing CCK concentrations (100 and $400 \mathrm{~nm}$ ), suppression became the predominant effect. The numbers in parentheses above each concentration indicate the number of experiments in each condition. $B$, Summary of effects of CCK on number of peaks (逐; \# peaks), oscillation amplitude (四; $A m p_{o s c}$ ), decay of oscillation $\left(\mathbb{\mathbb { N }} ; \tau_{\text {osc }}\right)$, and period of oscillation (冒; period) calculated from the autocorrelograms (see Materials and Methods for details). The values have been sorted by the overall effect and grouped by concentration. The histograms in $B i$ consist of only slices in which CCK produced a facilitation of the rhythmic activity, and have been grouped into $<100 \mathrm{nM}(20$ and $50 \mathrm{~nm})$ and $\geq 100 \mathrm{nM}$ CCK (100 and $400 \mathrm{~nm}$ ). Note the consistent increase in \# peaks, $\mathrm{Amp}_{\text {osc }}$, and $\tau_{\text {osc }}$, but no change in the period. Bii, Summary of the characteristics of the oscillatory activity for slices in which the rhythmic activity was suppressed by CCK. Note that \# peaks, Amposc, and $\tau_{\mathrm{osc}}$ are decreased by CCK, but the period remains unaltered. The ordinate is the ratio of $\mathrm{CCK} /$ pre-drug. * $p<0.05$.
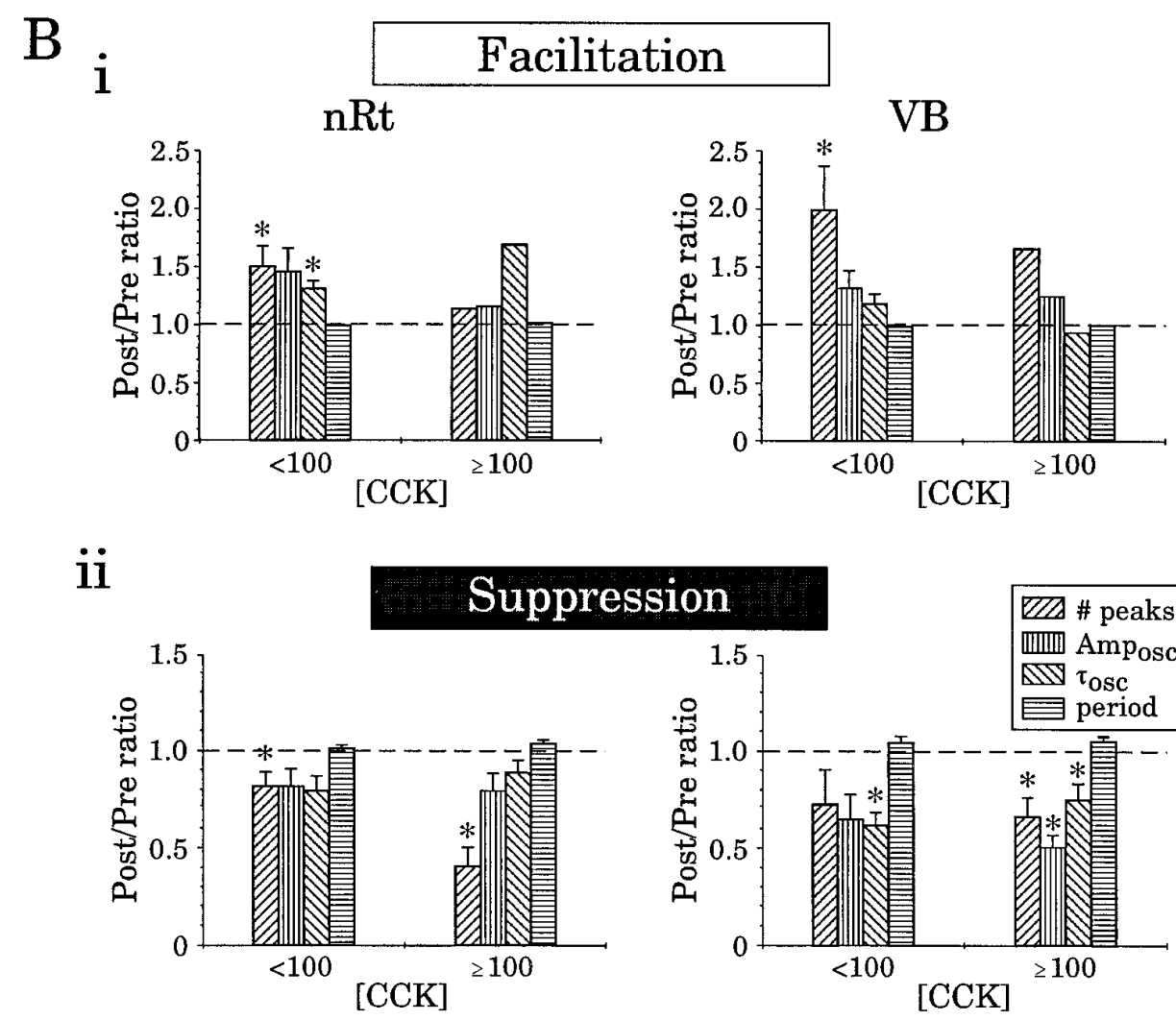

nearly all experiments (11 of 12) when higher CCK concentrations were used (100-400 nM; Fig. 5). This effect was accompanied by an increase in baseline discharge in nRt (Fig. 5Ai; compare Pre-drug vs $C C K$ ) but not in VB (Fig. $5 B i$ ), consistent with a selective CCK-induced depolarization of nRt neurons (Cox et al., 1995a). Note that although CCK increased the overall spike output of nRt neurons, the rhythmic discharge in VB was suppressed, suggesting that phasic nRt output is critical for maintenance of the rhythmic activity. The ratemeter plots of Figure 5, Aii and $B i i$, illustrate the magnitude and reversibility of the CCKinduced suppression of oscillatory activity. It was of interest that in four slices in which the overall CCK action was suppression of oscillations, there was a transient facilitation in the number of peaks of the oscillatory activity soon after the onset of CCK application or during washout of the peptide. The autocorrelograms of nRt activity in Figure 5Aiii show the complete suppression of distinct peaks during CCK perfusion and a robust increase in nonphasic unit discharge. In VB, both the number and amplitude of peaks were decreased in CCK with little effect on the nonphasic activity (Fig. 5Biii).

Data from a number of experiments are summarized in Figure $6 \mathrm{~A}$, which clearly illustrates the concentration-dependent biphasic effects of CCK on the oscillatory activity. Changes in the number of peaks and $\mathrm{Amp}_{\text {osc }}$ measurements obtained from the contour plots and autocorrelograms (see Materials and Methods) served as criteria for the overall action of CCK. The rhythmic activity was considered facilitated if both measures increased during CCK application (e.g., Fig. $4 B$ ), whereas reduction in both of these measures indicated a 
A

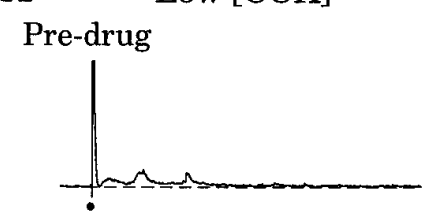

CCK (V $\left.\mathrm{V}_{\text {match }}\right)$

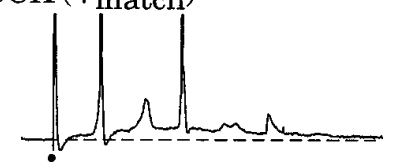

Post CCK

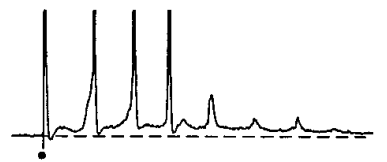

Wash

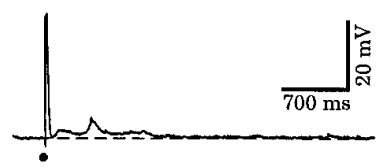

B $\quad$ High $[\mathrm{CCK}]$
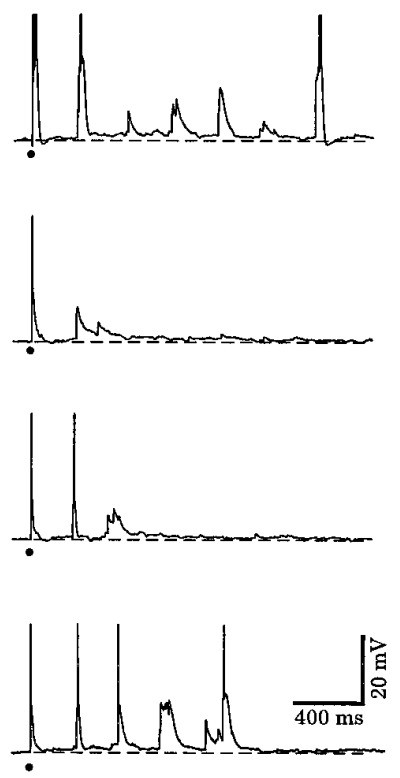

Figure 7. Pro- and antioscillatory actions of CCK8S on rhythmic EPSPs in nRt neurons. $A$, Intracellular recordings from an nRt neuron in which IC stimulation evokes rhythmic EPSPs (Pre-drug) that can lead to burst discharge (truncated spikes). The extracellular solution contained standard $\left[\mathrm{Mg}^{2+}\right](2$ $\mathrm{mM})$ and $10 \mu \mathrm{M} \mathrm{BMI}$. After manual repolarization of the membrane potential during the peak of a CCK8S (25 nM, bath-applied)-induced depolarization (5 $\mathrm{mV}$ ), the same stimulus evokes an increased number of rhythmic EPSPs $\left[C C K\left(V_{\text {match }}\right)\right]$. The enhanced rhythmic EPSPs persisted during washout of the peptide even as the membrane potential returned to within $2 \mathrm{mV}$ of the control membrane potential (Post CCK). After complete washout of the peptide (5 min), the evoked response returned to baseline conditions (Wash). $V_{\mathrm{m}}($ dashed line $)=-64 \mathrm{mV}$. B, Pre-drug, Stimulation of internal capsule evokes rhythmic EPSPs that persist for 6 cycles in another nRt neuron. This recording was made in standard physiological solution with no addition of BMI. During depolarization $(10 \mathrm{mV})$ induced by CCK8S (500 nM; $15 \mathrm{sec}$ bolus into input line; see Materials and Methods), the membrane potential is manually returned to baseline level and subsequent stimuli evoke a shorter lasting rhythmic response $\left[C C K\left(V_{\text {match }}\right)\right]$. The rhythmic activity was still suppressed when the membrane potential was within $2 \mathrm{mV}$ of control levels during washout of the peptide (Post CCK). After 4 min washout of the peptide, the synaptic response returns near pre-drug levels (Wash). $V_{\mathrm{m}}$ $($ dashed line $)=-63 \mathrm{mV}$.

CCK-induced suppression (e.g., Figs. 4A, 5). At low concentrations (20-50 nM), CCK was equally likely to produce facilitation or suppression. However, with increasing CCK concentrations (100 nM), suppression of the rhythmic activities became the predominant effect. At the highest CCK concentration tested ( $400 \mathrm{~nm})$, the intrathalamic oscillations were suppressed in all slices tested. There was no correlation between the overall CCK action on the oscillation (i.e., facilitation vs suppression) and the BMI concentration used to enhance the baseline oscillations. The characteristics calculated from the autocorrelograms (number of peaks, $A \mathrm{mp}_{\mathrm{osc}}, \tau_{\mathrm{osc}}$, and period; see Materials and Methods) are summarized in Figure $6 \mathrm{~B}$. Each of these different measures was generally consistent with the overall effect of CCK and reached statistical significance in many instances $(p<0.05$; $t$ test).

In the next series of experiments, we tested the action of CCK on the rhythmic EPSPs observed from whole-cell recordings of nRt neurons. In many of these experiments, IC stimulation evoked rhythmic EPSPs in nRt neurons in standard physiological solution without the addition of BMI (e.g., Fig. 7B). As expected

from extracellular recordings, low CCK concentrations (10-25 $\mathrm{nM}$ ) increased the number of rhythmic EPSPs in three of five neurons (Fig. 7A), an effect accompanied by a small membrane depolarization (range 4-10 $\mathrm{mV}$ ). The increased number of rhythmically occurring EPSPs was independent of CCK-induced depolarization of the individual neuron because it persisted after manual repolarization of the membrane potential during the peak of the CCK action [Fig. 7A, CCK $\left(V_{\text {match }}\right)$ ], and during early washout of the peptide, when the membrane potential had returned to within $2 \mathrm{mV}$ of the resting level (Fig. 7A, Post CCK). The finding that altering membrane potential of individual neurons did not influence the occurrence of the repetitive EPSPs suggests that the modification of the rhythmic activity is a result of a network effect. The rhythmic activity recovered to baseline conditions with further washout of the peptide (Fig. 7A, Wash). In three neurons, in which CCK produced suppression of the rhythms at the peak CCK depolarization, there was a transient facilitation of the rhythms during the washout of the peptide, similar to that observed in the extracellular recordings.

High CCK concentrations (100-1000 nM) reversibly suppressed the rhythmic EPSPs (Fig. $7 B ; n=12$ ), and also produced a substantially larger membrane depolarization (range, 8-19 mV). As with the lower CCK concentrations, reduction of the rhythmic activity in individual neurons was independent of membrane depolarization because it persisted after manual return of the membrane potential to resting level [Fig. $7 B, C C K\left(V_{\text {match }}\right)$ ], and after the membrane potential returned to within $2 \mathrm{mV}$ of the resting membrane potential after washout of the peptide (Fig. $7 B$, Post $C C K)$. Rhythmic EPSPs recovered to baseline levels after longer CCK wash (Fig. 7B, Wash).

\section{CCK modifies discharge mode of $\mathbf{n R t}$ neurons}

The generation and persistence of intrathalamic oscillations is dependent on the firing mode of $\mathrm{nRt}$ and relay thalamic neurons, which is in turn closely related to the membrane potential (Jahnsen and Llinás, 1984; Steriade and Deschênes, 1984; Steriade and Llinás, 1988; von Krosigk et al., 1993). Because the results described above indicated that CCK produces a concentration dependent effect on the rhythmic activity, we examined the action of CCK on membrane potential $\left(V_{\mathrm{m}}\right)$ and the discharge mode of nRt neurons in more detail in 15 neurons. At rest, nRt neurons were typically in a burst firing mode, such that either IC stimulation $(\cdot)$ or intracellular injection of depolarizing current evoked burst discharge (Fig. $8 A$ ). At the peak of the CCK-induced depolarization, both synaptic stimulation and the depolarizing current pulses evoked single spikes or tonic discharges (Fig. 8B, peak). Conversion of the firing mode from burst- to singlespike discharge was dependent on the magnitude of the depolarization because repolarization of the membrane potential to resting levels during the CCK-induced depolarization restored the neurons to bursting mode (Fig. $8 B, V_{\text {match }}$ ). After washout of the peptide, the neurons returned to burst firing mode (Fig. $8 C$ ). High CCK concentrations $(>100 \mathrm{~nm})$ that produced relatively large depolarizations (range, $8-27 \mathrm{mV})$ altered the firing mode in all cells tested $(n=11)$. Lower CCK concentrations (10-25 nM) produced smaller depolarizations $(n=8$; range, $3-22 \mathrm{mV})$. Changes in firing mode were observed only in those cases in which CCK induced a strong depolarization $(>14 \mathrm{mV} ; n=2)$. These data suggest that CCK, like other substances that directly depolarize thalamic neurons (e.g., NE and 5-HT; McCormick and Prince, 1988; McCormick and Wang, 1991), can alter the firing mode of the neurons though depolarization of the membrane potential.

We have shown previously that CCK reduces a $K_{\text {leak }}$ conduc- 


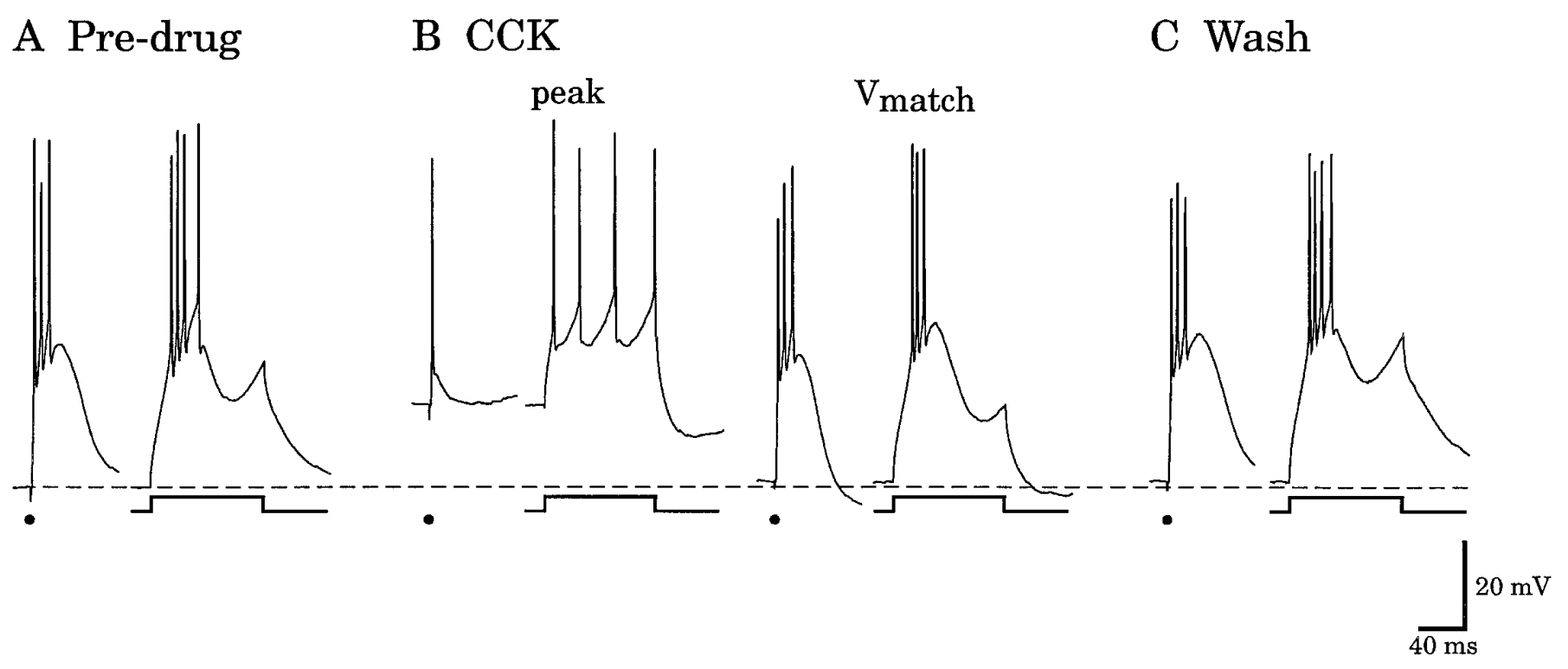

Figure 8. $\quad \mathrm{CCK}$ alters the firing mode of nRt neurons. A, Pre-drug, At resting membrane potential, either internal capsule stimuli (·) or intracellular depolarizing current pulses $(200 \mathrm{pA}, 100 \mathrm{msec})$ evoke burst discharge from an nRt neuron. $B$, CCK, During peak of the depolarization induced by application of $400 \mathrm{~nm} \mathrm{CCK8S,} \mathrm{same} \mathrm{stimuli} \mathrm{as} \mathrm{in} A$ evoked single spike discharge from the neuron ( peak). Repolarization of the membrane potential to resting levels restored the burst firing mode $\left(V_{\text {match }}\right)$. $C$, After washout of CCK8S, $V_{\mathrm{m}}$ returned to close to baseline level (dashed line) and both stimuli evoked burst discharge. $V_{\mathrm{m}}$ (dashed line $)=-73 \mathrm{mV}$.

tance in nRt neurons, resulting in a robust membrane depolarization associated with an increased input resistance (Cox et al., 1995a). The time course of the increased input resistance was generally equal to or longer than the membrane depolarization, and thus could lead to an enhanced excitability that may be independent of the depolarization. Consistent with this, we found that CCK could modify the responses of nRt neurons to subthreshold orthodromic stimulation with little alteration of membrane potential. As illustrated in Figure 9A, subthreshold EPSPs evoked by IC stimulation (Pre-drug) could evoke burst discharge during initial wash-in of a high CCK concentration (Fig. 9A: early), even though there was little change in the membrane potential $(<2 \mathrm{mV})$. At the peak CCK-induced depolarization, the firing mode of nRt neurons was altered from burst- to single-spike (Fig. 9A, peak). After manual repolarization of the membrane potential during the peak CCK-induced depolarization, subsequent stimuli evoked burst discharge (Fig. $9 A, V_{\text {match }}$ ). Thus, there was an enhancement of the subthreshold response observed in the pre-CCK condition that did not appear to be dependent on $V_{\mathrm{m}}$ (Fig. 9A: compare Pre-drug and $V_{\text {match }}$ ).

This response enhancement was explored further by testing the effect of low CCK concentrations (10 nM) on subthreshold EPSPs in seven nRt neurons. This concentration of CCK produced a small membrane depolarization (range, $2-8 \mathrm{mV}$ ) but increased the probability of suprathreshold spike discharges. As illustrated in Figure $9 B i$ (Pre-drug) and Bii, repeated stimuli $(0.1 \mathrm{~Hz})$ evoked a suprathreshold response in approximately $50 \%$ of trials. During CCK application, the stimuli evoked burst discharge in $100 \%$ of trials [Fig. 9Bi, CCK $(10 n \mathrm{M})$ ], and after several minutes of washout, the proportion of evoked subthreshold responses reverted to pre-CCK levels. The time course of the CCK-induced action on the subthreshold EPSP is illustrated in Figure 9Bii. These findings suggest that low CCK concentrations may increase the number of nRt neurons bursting and participating in the oscillatory activity, and thus increase the strength and duration of the evoked rhythmic activity, as illustrated by the extracellular data of Figure 4.

\section{CCK reduces inhibitory input to VB neurons}

Conversion of the firing mode of nRt neurons from bursts to single spikes by CCK would be expected to decrease the inhibitory output of nRt neurons onto VB neurons. In the next series of experiments, we directly assessed evoked IPSCs in VB neurons as a measure of nRt output. Extracellular stimulation of nRt evoked a multipeaked IPSC in VB neurons (Figs. 10 Ai,Aii). In most of these recordings, we substituted $\mathrm{Cs}^{+}$for $\mathrm{K}^{+}$in the intracellular solution and used a holding voltage near $0 \mathrm{mV}$ to increase the driving force on the IPSCs. Similar findings were observed with $\mathrm{K}^{+}$-containing electrodes $\left(V_{\text {hold }}=-40 \mathrm{mV}\right)$, although the multiple peaks of the IPSCs were not as distinct as in recordings with $\mathrm{Cs}^{+}$-containing electrodes. The interpeak intervals of the multipeaked IPSCs $(2.2-10.0 \mathrm{~ms} ; n=100$ intervals from 5 cells) roughly approximated the interspike interval of burst discharge in nRt neurons (2.3-9.5 ms; $n=100$ intervals from 5 cells; compare Fig. 8, nRt bursts, and Fig. 10 $A$, VB multipeaked IPSCs). In this first series of experiments, we determined whether the multipeak configuration in VB was due to burst firing of the nRt neurons at the somatic level or resulted from nonsynchronous transmitter release from multiple sites. We dissected $\mathrm{nRt}$ from the thalamic slice and stimulated the lateral edge of VB. Because nRt serves as the primary inhibitory innervation in rodent VB (Houser et al., 1980), the isolated VB was devoid of inhibitory neurons but still contained GABAergic fibers. Under these conditions, each stimulus should evoke a single volley in the cut nRt axons. Stimuli evoked single-peaked IPSCs (Figs. $10 B i, B i i ; n=9$ ), suggesting that nRt somata were required for the multipeaked IPSCs. Furthermore, in the intact slice, addition of ionotropic excitatory amino acid antagonists, CNQX $(20 \mu \mathrm{M})$ and dCPPene $(10 \mu \mathrm{M})$, reduced the multipeaked IPSC to a single peak (Fig. 10Aiii), indicating that the extracellular stimuli is activating excitatory afferents in nRt. The addition of CNQX and dCPPene produced no change in the IPSCs recorded in the isolated VB (Fig. 10Biii). In summary, these findings suggest that activation of excitatory 
A

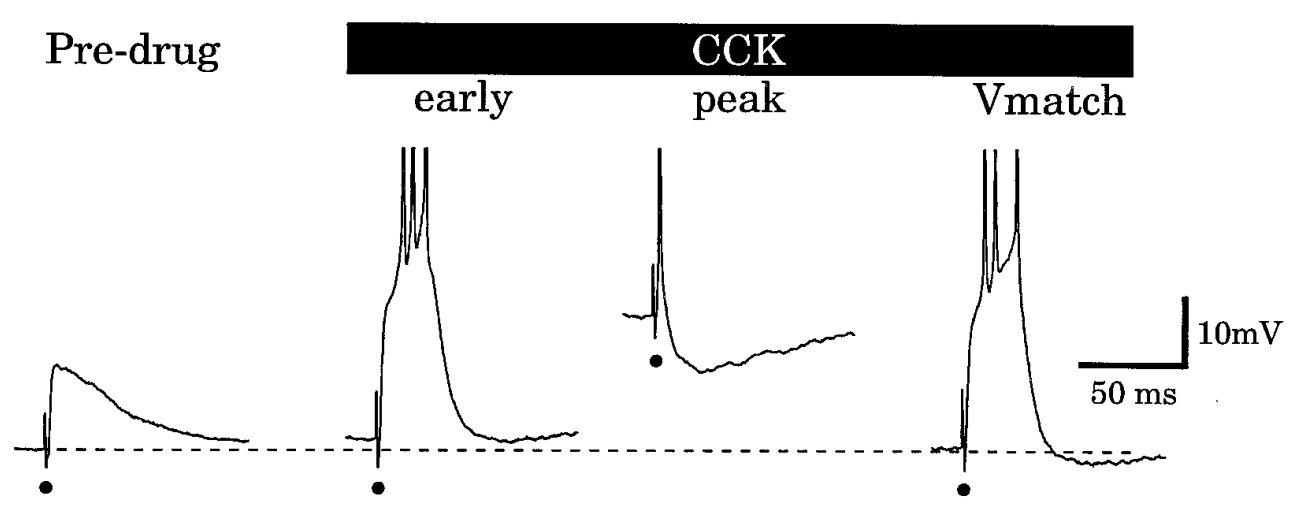

\section{$\mathrm{Bi}$}

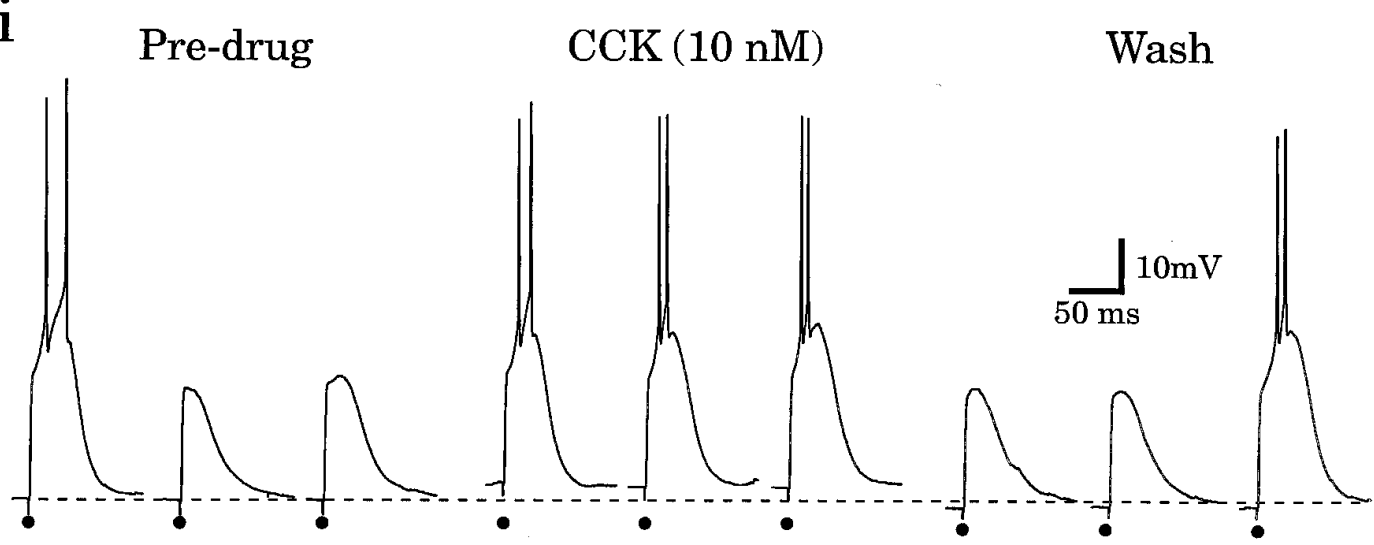

$\mathrm{Bii}$

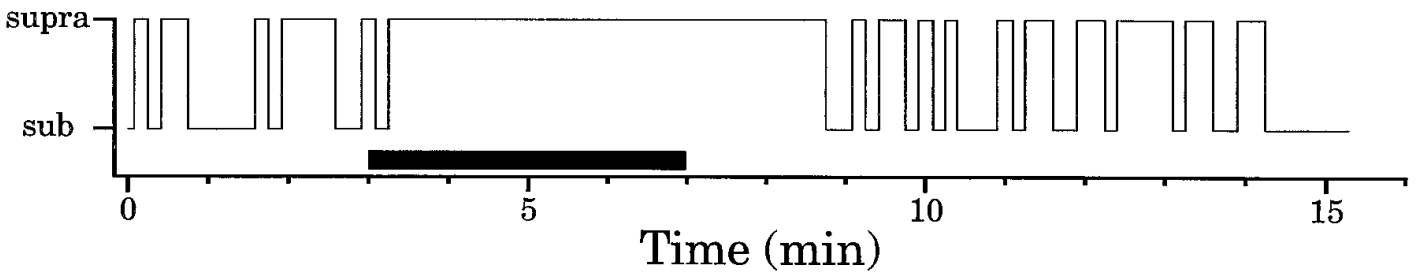

Figure 9. CCK increases probability of burst discharge to subthreshold stimulation. A, Pre-drug, Extracelluar IC stimulation evokes a subthreshold EPSP in a nRt neuron. $C C K$, early, During the initial depolarization produced at the onset of CCK8S application ( $3 \mu \mathrm{M}$, 10 sec bolus), IC stimuli evoked burst discharge (truncated spikes). At the peak of the CCK depolarization $(15 \mathrm{mV})$, the stimuli evoked single spike discharge (peak). After manual repolarization of the membrane potential to resting levels, the same stimuli that produced subthreshold responses in the control period (Pre-drug) evoked burst discharge $\left(V_{\text {match }}\right)$. Dashed line, $V_{\mathrm{m}}=-69 \mathrm{mV}$. Bi, In a different $\mathrm{nRt}$ neuron, extracellular stimulation evoked subthreshold EPSPs in $\sim 50 \%$ of trials (Pre-drug; three consecutive responses). After wash-in of $10 \mathrm{nM}$ CCK8S, which produced a $2 \mathrm{mV}$ peak depolarization, the same stimuli evoked burst discharges $[C C K(10 \mathrm{~nm})]$. The synaptic response returned to baseline condition after washout of CCK $($ Wash $)$. Dashed line, $V_{\mathrm{m}}=-68 \mathrm{mV}$. Bii, This plot illustrates the time course of the CCK-induced alteration of the subthreshold responses of the experiment in Bi. Bath application of CCK (10 nM) occurred during the solid bar. Before CCK, extracellular stimulation evoked either EPSPs (sub) or EPSPs that led to burst discharge (supra) in nearly equal proportions. In CCK, all evoked responses were suprathreshold and returned toward baseline levels after several minutes of washout.

afferents onto nRt neurons produced burst discharge in these cells, and thus evoked multipeaked IPSCs in VB neurons.

Because the multipeaked IPSCs in VB are reflective of the firing mode of the nRt neurons, and CCK was found to alter the firing mode of nRt neurons (Fig. 8), we tested several concentrations of CCK on the IPSCs. High CCK concentrations (>100 nM) altered the configuration of the IPSCs from multiple peaks to a single peak (Fig. $11 B)$ and significantly reduced the integrated areas of IPSCs by an average of $62.7 \pm 20.3 \%(n=15$; paired $t$ test, $p<0.05)$. Although lower CCK concentrations also significantly reduced IPSC areas (average $27.8 \pm 16.3 \% ; n=3$ ), the multipeak configuration of the IPSCs was unchanged, suggesting that the firing mode of only a subpopulation of neurons was altered (Fig. 11A). The summarized data (Fig. 11C) indicate that CCK produced a significant dosedependent suppression of the IPSC-integrated area.

\section{DISCUSSION}

Our findings indicate that the neuropeptide CCK has a strong influence on intrathalamic rhythmic activities. Neuropeptides have been shown to produce lasting alterations in neuronal network functions in various invertebrate and vertebrate preparations, which may be associated with behavioral modifications (for review, see Harris-Warrick and Marder, 1991; Kupfermann, 1991). Because intrathalamic oscillations are 

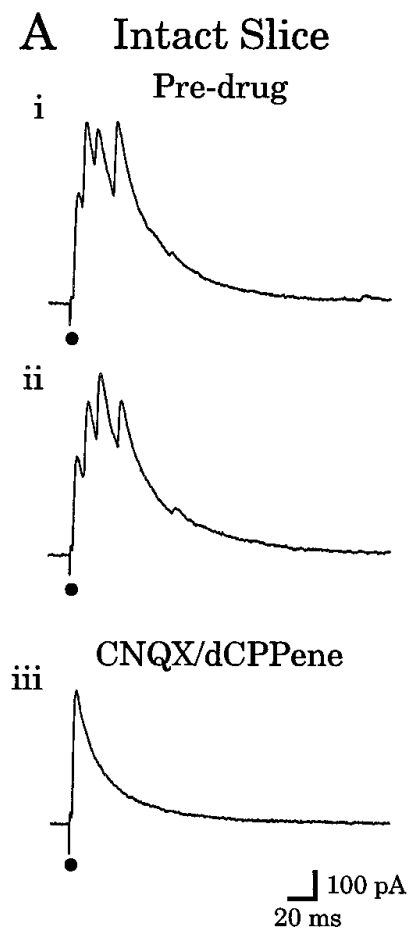

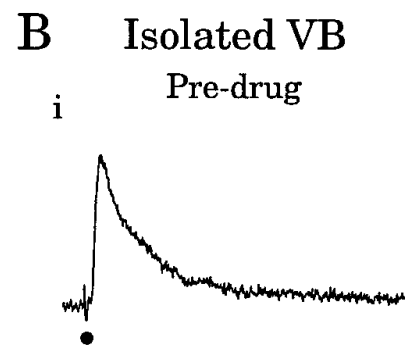

ii
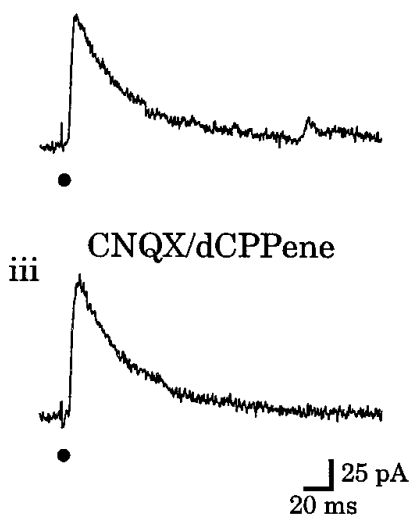

A

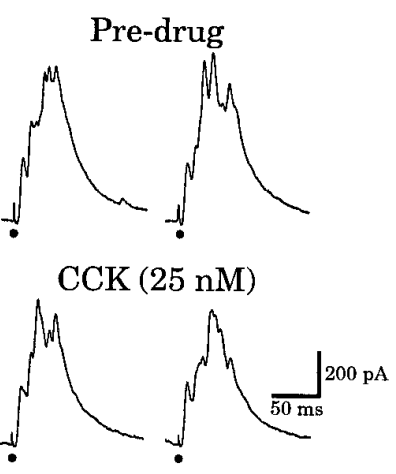

B

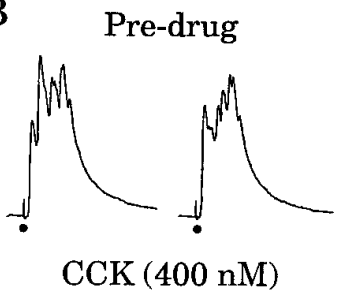

C
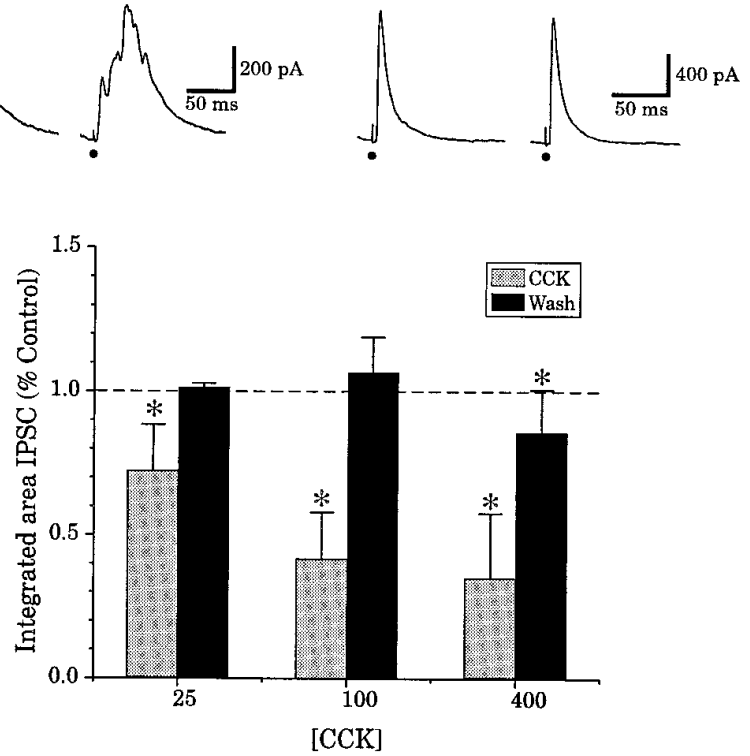

Figure 11. CCK alters the multipeaked configuration of IPSCs in VB neurons. A, Pre-drug, In the intact thalamic slice, extracellular stimulation of $\mathrm{nRt}$ evokes a multipeaked IPSC in a VB neuron $\left(V_{\text {hold }}=0\right.$ $\mathrm{mV}$ ). Each pair of responses illustrated is evoked by consecutive stimuli to illustrate the consistency of the configuration of the multiple peaks of the IPSC. CCK $(25 n M)$, During application of CCK8S, the multipeaked configuration of the IPSC is unchanged, although the total charge of the IPSC (Integrated area) is reversibly attenuated by CCK8S. $B$, Pre-drug, In a different VB neuron, nRt stimulation evoked a multipeaked IPSC. $C C K(400 \mathrm{~nm})$, When the slice is exposed to $400 \mathrm{~nm}$ CCK, a concentration that suppresses oscillatory activity, the IPSC is reduced to a single peak. $C$, Summary of the effect of various CCK concentrations on mean charge of IPSCs. The ordinate is the ratio of mean integrated areas of IPSCs in CCK/control (Pre-drug). The effects of CCK and wash were calculated as ratios relative to pre-drug values. Data are plotted as mean $\pm \mathrm{SD}\left({ }^{*} p<0.05\right)$.

The effects of CCK on intrathalamic oscillations are concentration dependent. Low concentrations of CCK suppressed rhythmic interactions between $\mathrm{nRt}$ and VB in $43 \%$ of experiments, whereas rhythmic activities were facilitated in the remaining. We speculate that application of lower concentrations of exogenous CCK may more closely approximate the level of modulation that occurs in vivo during thalamocortical circuit activities. With increasing CCK concentrations, suppression of rhythmic activity became the dominant action. Our working hypothesis is that the predominant suppression of rhythmic activity at higher CCK concentrations results from a strong depolarization of $\mathrm{nRt}$ neurons, which alters their firing mode from a resting burst- to single-spike discharge mode. This explanation is supported by direct recordings of the effects of CCK on nRt neurons and the resulting change of inhibitory output onto VB cells. Bath-applied CCK induces a concentration-dependent, long-lasting depolarization of $\mathrm{nRt}$ neurons by suppressing a $\mathrm{K}^{+}$leak conductance, without altering the membrane potential or input resistance of VB neurons (Cox et al., 1995a). Intracellular recordings of the rhythmic activity in nRt neurons indicate that suppression of this activity was associated with a larger CCK-induced depolarization than that observed in other $\mathrm{nRt}$ cells, the rhythmic activity of which was facilitated. High CCK concentrations, which produced robust membrane depolarizations, clearly altered the spike discharge characteristics of the nRt neurons from burst- to single-spike firing mode (Fig. 8). Lower CCK concentrations that also produced relatively large depolarizations likewise altered the firing characteristics of these neurons. However, if CCK produced only a small membrane depolarization, the firing mode of the cell remained unchanged.

Because GABA-containing neurons of $\mathrm{nRt}$ provide the major source of inhibitory innervation of VB in rodents, a decrease in spike output of nRt neurons would reduce the inhibitory drive onto VB neurons. Intracellular recordings from VB neurons showed that nRt stimulation evoked multipeaked IPSCs, consistent with the burst output of the nRt cells. CCK, at concentrations that suppressed the rhythmic activity, changed the configuration of the IPSCs from multiple peaks to a single peak, a finding consistent with the CCK-induced alteration of nRt firing modes from burst to single spike. This decreased inhibitory current 
would reduce the probability of burst output from VB, and thus dampen the rhythmic activity.

The pro-oscillatory actions of CCK are more difficult to account for on the basis of a similar action of CCK. The increased strength and duration of the rhythmic activity likely occurs as a consequence of recruitment of additional nRt neurons that were subthreshold for burst generation before CCK exposure (Fig. 9B). CCK could have a biphasic action that is dependent on the resting membrane potential of the aggregate of $\mathrm{nRt}$ neurons. If portions of the latter were significantly hyperpolarized at rest, synaptic inputs might be subthreshold for evoking bursts of spikes, even though the low threshold $\mathrm{Ca}^{2+}$ current $\left(I_{\mathrm{T}}\right)$ underlying the burst behavior might be fully deinactivated (Coulter et al., 1989; Huguenard and Prince, 1992). Under these conditions, small depolarizations produced by low concentrations of CCK could increase the population of nRt neurons engaged in burst behavior by allowing synaptically evoked LTSs to reach spike threshold, thus facilitating oscillatory activity in the circuit. However, the increased excitability of nRt neurons was observed even when CCK produced little obvious membrane depolarization (Fig. 9). The cellular mechanisms underlying this facilitation of oscillatory behavior are not clear. One possibility is that low concentrations of CCK induce increases of input resistance and membrane depolarization at sites that are electrotonically distant from the soma (Cox et al., 1995a) and undetectable with somatic recordings. Enhanced EPSPs might result, leading to more effective triggering of LTSs and bursts. Alternatively, enhanced EPSPs could occur by presynaptic actions of CCK, as suggested in other CNS regions (Delfs and Dichter, 1985; Migaud et al., 1994). Subtle effects on the properties of $I_{\mathrm{T}}$ channels could also potently influence oscillatory activities in the network (Huguenard and Prince, 1994).

\section{Functional significance of CCK effects on intrathalamic oscillations}

The intimate reciprocal synaptic connectivity between nRt and the adjacent thalamic relay nucleus, together with the intrinsic properties of the involved neuronal populations, is the basis of intrathalamic rhythmic activities that have been associated with sleep spindles, slow-wave sleep, and the $3 \mathrm{~Hz}$ rhythms observed during absence epileptic seizures (Steriade and Llinás, 1988; Steriade et al., 1993; von Krosigk et al., 1993; Huguenard and Prince, 1994; Bal et al., 1995). Maintenance or disruption of these rhythmic activities is dependent on the firing mode of thalamic neurons.

Although the fundamental mechanisms underlying the maintenance of thalamic oscillatory activity have been studied extensively, the mechanisms underlying the initiation, cessation and modulation of this rhythmic activity, have received less attention. Our data indicate that CCK can either enhance or dampen intrathalamic rhythmic activity. Other neuromodulators, such as ACh, NE, and 5-HT, alter the firing mode of thalamic neurons by modifying the membrane potential of these neurons (McCormick and Prince, 1987; McCormick and Prince, 1988; McCormick and Wang, 1991). Similarly, the overall effect of CCK on a given nRt neuron depends on the initial membrane potential of the neuron and the strength of depolarization. Unlike other neuromodulators such as ACh and NE, CCK is contained within thalamocortical and corticothalamic neurons (Ingram et al., 1989; Burgunder and Young, 1990; Schiffmann and Vanderhaeghen, 1991), and thus may serve as an intrinsic modulator of thalamic circuits.

These two different sites of origin for CCK-containing afferents both send axon collaterals into nRt (Jones, 1985). On the basis of evidence suggesting that high-frequency spike discharges may be optimal for inducing peptide release at other sites (Bartfai et al., 1988), we speculate that the influence of CCK might normally be most prominent in $\mathrm{nRt}$ during periods of repetitive burst discharge of VB relay cells. Considering the long duration of CCK action (Cox et al., 1995a), release of the peptide during each cycle of the thalamic burst rhythm could induce a slow depolarization of nRt neurons such as has been observed in vivo (Steriade and Deschênes, 1984; Domich et al., 1986; Contreras and Steriade, 1996) and in vitro (Fig. 7). During thalamic spindle sequences in vivo, cat nRt neurons generate slow $(0.1-0.2 \mathrm{~Hz})$ depolarizations that may have a role in terminating rhythmic burst activity (Mulle et al., 1986). With increasing depolarization, the firing mode of nRt neurons would shift from burst to tonic firing, and thus dampen the rhythmic activity. It has been suggested that release of peptide (somatostatin from nRt cells, Mulle et al., 1986) could have a role in modifying such slow oscillations. A modulatory role for CCK in rat thalamus has also been suggested on the basis of the dense distribution of CCK binding sites in nRt (Pelaprat et al., 1987).

Release of CCK in nRt by corticothalamic neurons might have quite different consequences. A synchronous high-frequency discharge, such as occurs during focal cortical epileptiform events (Gutnick and Prince, 1974), could provide the necessary stimulus for CCK release, resulting in depolarization and onset of regular spiking or burst discharge of a group of $\mathrm{nRt}$ neurons in a localized sector of the nucleus, depending on their resting membrane potential and the intensity of corticothalamic activity. Because the axonal arborizations of a subgroup of $\mathrm{nRt}$ neurons project focally within VB and may possess a high density of axonal swellings (Pinault et al., 1995; Cox et al., 1996), strong depolarization of a few nRt neurons may release sufficient amounts of GABA to strongly hyperpolarize relay neurons, resulting in generation of rhythmic activity. Under the conditions of our experiments, burst discharge from a single nRt neuron can activate a sufficient number of VB neurons to reexcite the nRt cell and trigger burst discharge (C.L. Cox, J.R. Huguenard and D.A. Prince, unpublished observations). In the ferret, a single burst of an individual perigeniculate neuron can initiate a spindle wave but not the slow rhythmic activity (Kim et al., 1995). Knowledge of the degree of divergence and convergence of the connectivity between $\mathrm{nRt}$ and the relay nucleus, as well as the physiological strength of corticothalamic and intrathalamic synaptic inputs to each nucleus, are thus important for understanding the minimal cellular substrate required to initiate and generate these intrathalamic rhythms.

\section{REFERENCES}

Albrecht D, Müller R, Zippel U, Gabriel H-J, Henklein P, Davidowa H (1994) Cholecystokinin-induced activity changes of dorsal lateral geniculate neurons in rats. Neuroscience 59:953-960.

Bal T, von Krosigk M, McCormick DA (1995) Role of the ferret perigeniculate nucleus in the generation of synchronized oscillations in vitro. J Physiol (Lond) 483:665-685.

Bartfai T, Iverfeldt K, Fisone G, Serfözö P (1988) Regulation of the release of coexisting neurotransmitters. Annu Rev Pharmacol Toxicol 28:285-310.

Battaglia G, Colacitti C, Bentivoglio M (1992) The relationship of calbindin-containing neurons with substance $\mathrm{P}$, leu-enkephalin and cholecystokinin fibres: an immunohistochemical study in the rat thalamus. J Chem Neuroanat 5:453-464.

Blanton MG, Lo Turco JJ, Kriegstein AR (1989) Whole cell recording from neurons in slices of reptilian and mammalian cerebral cortex. J Neurosci Methods 30:203-210.

Burgunder J-M, Young III WS (1990) Cortical neurons expressing the cholecystokinin gene in the rat: distribution in the adult brain, ontogeny, and some of their projections. J Comp Neurol 300:26-46. 
Burgunder J-M, Young III WS (1992) Expression of cholecystokinin and somatostatin genes in the human thalamus. J Comp Neurol 324:14-22.

Contreras D, Steriade M (1996) Spindle oscillations in cats: the role of corticothalamic feedback in a thalamically generated rhythm. J Physiol (Lond) 490:159-179.

Coulter DA, Huguenard JR, Prince DA (1989) Calcium currents in rat thalamocortical relay neurones: kinetic properties of the transient, low-threshold current. J Physiol (Lond) 414:587-604.

Cox CL, Huguenard JR, Prince DA (1995a) Cholecystokinin depolarizes rat thalamic reticular neurons by suppressing a $\mathrm{K}^{+}$conductance. J Neurophysiol 74:990-1000.

Cox CL, Huguenard JR, Prince DA (1995b) Cholecystokinin modulates intrathalamic oscillations. Soc Neurosci Abstr 21:404.2.

Cox CL, Huguenard JR, Prince DA (1996) Hetereogenous axonal arborizations of rat thalamic reticular neurons in the ventrobasal nucleus. J Comp Neurol 366:416-430.

Davidowa H, Albrecht D, Gabriel H-J, Zippel U (1995) Cholecystokinin affects the neuronal discharge mode in the rat lateral geniculate body. Brain Res Bull 36:533-537.

Delfs JR, Dichter MA (1985) Cholecystokinin-octapeptide enhances synaptic activity in neurons cultured from rat cerebral cortex. Peptides 6:139-144.

Deschênes M, Paradis M, Roy JP, Steriade M (1984) Electrophysiology of neurons of lateral thalamic nuclei in cat: resting properties and burst discharges. J Neurophysiol 51:1196-1219.

Domich L, Oakson G, Steriade M (1986) Thalamic burst patterns in the naturally sleeping cat: a comparison between cortically-projecting and reticularis neurones. J Physiol (Lond) 379:429-449.

Edwards FA, Konnerth A, Sakmann B, Takahashi T (1989) A thin slice preparation for patch clamp recordings from neurons of the mammalian central nervous system. Pflügers Arch 414:600-612.

Gray CM, Singer W (1989) Stimulus-specific neuronal oscillations in orientation columns of cat visual cortex. Proc Natl Acad Sci USA 86:1698-1702.

Gutnick MJ, Prince DA (1974) Effects of projected cortical epileptiform discharges on neuronal activities in cat VPL. I. Interictal discharge. J Neurophysiol 37:1310-1327.

Harris-Warrick RM, Marder E (1991) Modulation of neural networks for behavior. Annu Rev Neurosci 14:39-57.

Hendry SHC, Jones EG, DeFelipe J, Schmechel D, Brandon C, Emson PC (1984) Neuropeptide-containing neurons of the cerebral cortex are also GABAergic. Proc Natl Acad Sci USA 81:6526-6530.

Houser CR, Vaughn JE, Barber RP, Roberts E (1980) GABA neurons are the major cell type of the nucleus reticularis thalami. Brain Res 200:341-354.

Hökfelt T, Fried G, Hansen S, Holets V, Lundberg JM, Skirboll L (1986) Neurons with multiple messengers-distribution and possible functional significance. Prog Brain Res 65:115-137.

Hu B, Steriade M, Deschênes M (1989a) The effects of brainstem peribrachial stimulation on perigeniculate neurons: the blockade of spindle waves. Neuroscience 31:1-12.

Hu B, Steriade M, Deschênes M (1989b) The effects of brainstem peribrachial stimulation on neurons of the lateral geniculate nucleus. Neuroscience 31:13-24.

Hubel DH (1960) Single unit activity in lateral geniculate body and optic tract of unrestrained cats. J Physiol (Lond) 150:91-104.

Huguenard JR, Prince DA (1992) A novel T-type current underlies prolonged $\mathrm{Ca}^{2+}$-dependent burst firing in GABAergic neurons of rat thalamic reticular nucleus. J Neurosci 12:3804-3817.

Huguenard JR, Prince DA (1994) Intrathalamic rhythmicity studies in vitro: nominal t-current modulation causes robust antioscillatory effects. J Neurosci 14:5485-5502.

Hunt CA, Seroogy KB, Gall CM, Jones EG (1987) Cholecystokinin innervation of rat thalamus, including fibers to ventroposterolateral nucleus from dorsal column nuclei. Brain Res 426:257-269.

Ingram SM, Krause RG, Baldino FJ, Skeen LC, Lewis ME (1989) Neuronal localization of cholecystokinin mRNA in the rat brain by using in situ hybridization histochemistry. J Comp Neurol 287:260-272.
Jahnsen H, Llinás R (1984) Electrophysiological properties of guinea-pig thalamic neurones: an in vitro study. J Physiol (Lond) 349:205-226.

Jones EG (1985) The thalamus. New York: Plenum.

Kim U, Bal T, McCormick DA (1995) Spindle waves are propagating synchronized oscillations in the ferret LGNd in vitro. J Neurophysiol 74:1301-1323.

Kupfermann I (1991) Functional studies of cotransmission. Physiol Rev 71:683-732.

Lundberg JM, Hökfelt T (1983) Coexistence of peptides and classical neurotransmitters. Trends Neurosci 6:325-333.

McCormick DA, Prince DA (1987) Actions of acetylcholine in the guinea-pig and cat medial and lateral geniculate nuclei, in vitro. J Physiol (Lond) 392:147-165.

McCormick DA, Prince DA (1988) Noradrenergic modulation of firing pattern in guinea pig and cat thalamic neurons, in vitro. J Neurophysiol 59:978-996.

McCormick DA, Wang Z (1991) Serotonin and noradrenaline excite GABAergic neurones of the guinea pig and cat thalamic reticular nucleus. J Physiol (Lond) 442:235-255.

Migaud M, Roques BP, Durieux C (1994) Effects of cholecystokinin octapeptide and BC 264, a potent and selective CCK-B agonist on aspartate and glutamate release from rat hippocampal slices. Neuropharmacology 33:737-743.

Mulle C, Madariaga A, Deschêanes M (1986) Morphology and electrophysiological properties of reticularis thalami neurons in cat: in vivo study of a thalamic pacemaker. J Neurosci 6:2134-2145.

Ogawa R, Itoh K, Kaneko T, Mizuno N (1989) Co-existence of vasoactive intestinal polypeptide (VIP)- and cholecystokinin (CCK)-like immunoreactivities in thalamocortical neuron in the ventrolateral nucleus of the rat. Brain Res 490:152-156.

Pelaprat D, Peschanski M, Broer Y, Besson JM, Rowues BP (1987) Postsynaptic receptors for cholecystokinin in the thalamic reticular nucleus: a possible modulatory system for sensory transmission. Neurosci Lett 80:16-20.

Pinault D, Bourassa J, Deschênes M (1995) The axonal arborization of single thalamic reticular neurons in the somatosensory thalamus of the rat. Eur J Neurosci 7:31-40.

Schiffmann SN, Vanderhaeghen J-J (1991) Distribution of cells containing mRNA encoding cholecystokinin in the rat central nervous system. J Comp Neurol 304:219-233.

Senatorov VV, Trudeau VL, Hu B (1995) Expression of cholecystokinin mRNA in corticothalamic projecting neurons: a combined fluorescence in situ hybridization and retrograde tracing study in the ventrolateral thalamus of the rat. Mol Brain Res 30:87-96.

Steriade M, Deschênes M (1984) The thalamus as a neuronal oscillator. Brain Res Rev 8:1-63.

Steriade M, Llinás R (1988) The functional states of the thalamus and the associated neuronal interplay. Physiol Rev 68:649-742.

Steriade M, McCormick DA, Sejnowski TJ (1993) Thalamocortical oscillations in the sleeping and aroused brain. Science 262:679-685.

Stuart GJ, Dodt HU, Sakmann B (1993) Patch-clamp recordings from the soma and dendrites of neurons in brain slices using infrared video microscopy. Eur J Physiol 423:511-518.

von Krosigk M, Bal T, McCormick DA (1993) Cellular mechanisms of a synchronized oscillation in the thalamus. Science 261:361-364.

Warren RA, Agmon A, Jones EG (1994) Oscillatory synaptic interactions between ventroposterior and reticular neurons in mouse thalamus in vitro. J Neurophysiol 72:1993-2003.

Williams D (1953) A study of thalamic and cortical rhythms in petit mal. Brain 76:50-69.

Xu M, Aiuchi T, Nakaya K, Arakawa H, Maeda M, Tsuji A, Kato T, Takeshige C, Nakamura Y (1990) Effect of low-frequency electric stimulation on in vivo release of cholecystokinin-like immunoreactivity in medial thalamus of conscious rat. Neurosci Lett 118:205-207.

Zarbin MA, Innis RB, Wamsley JK, Snyder SH, Kuhar MJ (1983) Autoradiographic localization of cholecystokinin receptors in rodent brain. J Neurosci 3:877-906. 